\title{
Anti-de-Sitter spacetime and its uses
}

\author{
G W Gibbons \\ D.A.M.T.P., \\ Cambridge University, \\ Silver Street, \\ Cambridge CB3 9EW, \\ U.K.
}

October 7, 2011

\begin{abstract}
This is a pedagogic account 1 of some of the global properties of Antide-Sitter spacetime with a view to their application to the AdS/CFT correspondence. Particular care is taken over the distinction between Anti-de-Sitter and it's covering space. It is argued that it is the former which is important.
\end{abstract}

\section{Introduction}

Because it is among the simplest of curved spacetimes, $n$-dimensional Anti-deSitter spacetime (AdS) has been of continuing interest to relativists. It has, since the earliest times of our subject, provided a test bed and a source of simple examples on which to try out novel ideas and spacetime concepts, both classical and quantum. It is a remarkable feature of the current search for a reformulation of the entire basis of theoretical physics, often referred to as Mtheory, that of many of those older speculations find a natural home in, and have relevance for, present day efforts. This point will be amply illustrated in what follows.

Because it is homogeneous and has a large isometry group, $S O(n-1,2)$, $A d S_{n}$ is the natural arena for enquiring to what extent the (Wignerian) grouptheoretic ideas underlying relativistic quantum mechanics and quantum field theory in Minkowski spacetime $\mathbb{E}^{n-1,1}$, with isometry group the Poincaré group $E(n-1,1)$, extend to other spacetimes. Similar remarks apply to ideas about energy momentum and angular momentum conservation. The definitions of the

\footnotetext{
${ }^{1}$ Written version of lectures given at 2nd Samos Meeting held at at Pythagoreon, Samos, Greece, 31 August - 4 September 1998 and published as Anti-de-Sitter spacetime and its uses, in Mathematical and Quantum Aspects of Relativity and Cosmology. Proceedings of the 2nd Samos Meeting on Cosmology, Geometry and Relativity, S Cotsakis and G W Gibbons eds, Lecture Notes in Physics $\mathbf{5 3 7}$ (2000)
} 
ADM mass in General Relativity and the question of its' positivity, which are closely linked, via Noether's theorem, to the properties of the isometry group [48, .

When quantizing field theories we often seek a background or "ground state" around which to perform a perturbation expansion and $A d S_{n}$ together with deSitter spacetime, $d S_{n}$ with isometry group $S O(n, 1)$, and Minkowski spacetime exhaust the list of maximally symmetric ground states. While de-Sitter spacetime arises naturally in studies of inflation, Anti-de-Sitter spacetime arises as the natural ground state of gauged supergravity theories.

We can regard flat space as a limit of the de-Sitter spacetimes as the cosmological constant goes to zero. In the process the isometry group undergoes a Wigner-Inönü 2 contraction to the Poincaré group. It is interesting to note therefore that a simple Lie-algebra cohomology argument gives a converse: these are the only isometry groups that may be obtained in this way $[3$.

A major topic of interest in quantum gravity is the extent to which the global and topological properties of spacetime, such as the existence of closed timelike curves (CTC's), spatial compactness etc, feed into the quantum theory. Indeed there is a more basic question: how do geometrical and spacetime concepts themselves translate into quantum mechanical language. In the case of deSitter and Anti-deSitter spacetimes, with space and time topology $S^{n-1} \times \mathbb{R}$ and $\mathbb{R}^{n-1} \times S^{1}$ respectively, and because of the high degree of symmetry, these questions may frequently be translated into group-theoretic language which may then admit a simple group-theoretic answer. In this connection it is essential to be aware of the many important differences between the properties of the compact Lie groups which particle physicists are most often familiar with and those of the isometry groups of Lorentzian spacetimes which are almost always non-compact 2 .

Currently a great deal of attention has been focussed on Anti-de-Sitter spacetimes because (multiplied by a sphere) they arise as the near horizon geometry of the extreme black holes and extreme p-branes which play such an an essential role in our understanding of M-theory. This has led to Maldacena's AdS/CFT correspondence conjecture which places AdS and indeed Euclidean Quantum gravity at the centre stage. In an interesting parallel and closely linked development, the mass and event horizon area properties of topologically non-trivial black holes, which can only arise in Anti-de-Sitter backgrounds have also attracted a great deal of interest recently.

In the notes which follow, I shall argue that it is fruitful if not essential to view these recent problems, like the former ones with the correct global perspective and that if one does so one arrives at what at first may appear to be some surprising and counter-intuitive conclusions. For that reason, and in view of the audience's interests, I shall be concentrating on the basic geometrical and group theoretic descriptions rather on the more technical details concerning supersymetry, supergravity and supertstring theory. For an earlier account with

\footnotetext{
${ }^{2}$ Lorentzian Taub-NUT spacetimes with isometry group $S U(2)$ or $S O(3)$ are an interesting exception
} 
more emphasis on the supergravity applications the reader is referred to [5]. One striking feature, which is especially appropriate for this meeting is that much of the discussion can be couched in the simple geometrical terms which would have been accessible to scientific workers in this city, and possibly on this very spot, two and a half millennia ago.

\section{M-Theory}

By way of motivation, recall that whatever it finally turns out to be, M-theory is a theory about $p$-branes, that is extended objects with $p$ spatial dimensions moving in some higher dimensional spacetime, usually eleven dimensions. Thus $p=0$ are point particles, $p=1$ are strings $p=2$ are membranes etc. The case $p=-1$ arises as "instantons".

\subsection{Levels of Description}

Currently we have various levels of description at various levels of approximation for dealing with branes in M-theory.

- As D-branes, that is as the end points of fundamental or F-strings subject to Dirichlet boundary conditions. At this level it is believed that one may use the techniques of two-dimensional conformal field theory (CFT) to give a fully quantum mechanical treatment.

- As "soliton" solutions of classical supergravity theories. This is the "heavy" brane approximation which takes into account their self gravity and is believed to be applicable in the semi-classical approximation when a large number, $N$, of light branes sit on top of one another. The solutions one starts with are typically, static, have extreme Killing horizons and are BPS, which means that they admit some Killing spinor fields of the associated supergravity theory.

- As classical solutions of a Dirac-Born-Infeld lagrangian describing a "light" brane, thought of as a $(p+1)$-dimensional timelike submanifold $\Sigma_{p+1}$ moving in a fixed spacetime background $M$. The equations of motion are a generalization of the standard equations for a minimal submanifold because in addition to the embedding map $x: \Sigma_{p+1} \rightarrow M$ (which provides scalar fields on the world volume $\Sigma_{p+1}$ ) each D-brane carries an abelian gauge field $A_{\mu}$ which may be viewed as $U(1)$ connection on a bundle over $\Sigma_{p+1}$. From the string theory standpoint, this vector field is associated with a open string of almost vanishing length, beginning and ending ending on the D-brane. Because the string has almost vanishing length it has almost vanishing energy and gives rise to a "light state" associated with the massless gauge field $A_{\mu}$. 
Strictly speaking the list given above does not exhaust all current brane descriptions because it omits the M5-brane action. However the details of the M5-brane action will not play an essential role in the future discussion.

\subsection{Symmetry Enhancement}

If one has $N$ branes one has has $N U(1)$ gauge fields. Now as the branes coalesce one might have supposed one would get a description in which one has a $U(1)^{N}$ gauge theory over the coalesced brane world volume $\bar{\Sigma}_{p+1}$. However from the string standpoint it is clear that $N(N-1)$ extra "light states" appear associated with strings of almost vanishing length beginning on one of the $N$ strings and ending on another. This gives rise to a total of $N^{2}$ massless gauge fields on $\bar{\Sigma}_{p+1}$. Again one might have supposed that this would give rise to a description in which one has a $U(1)^{N^{2}}$ gauge theory on $\bar{\Sigma}_{p+1}$. However, in a way which so far has only been understood in detail using conformal field theory, a process of non-abelian symmetry enhancement is believed to occur and the resultant gauge group becomes non-abelian, and in fact $U(N)$. The $U(1)$ factor is associated to the centre of mass motion of the D-brane.

\subsection{Killing spinors}

A supersymmetric solution of a supergravity theory is one admitting one or more spinor fields $\epsilon$ satisfying

$$
\nabla \epsilon+N \epsilon=0
$$

where $\nabla$ is the Levi-Civita connection and $N$ is a Clifford algebra valued oneform. The form of $N$ depends on the details of the supergravity theory concerned. If $N=0$ then a Killing spinor must be covariantly constant. This leads to the study of those holonomy groups which stabilize a spinor. The examples best known to relativists are the pp-waves. In $A d S_{n}$ one has

$$
N_{\alpha}= \pm \frac{1}{2 R} \gamma_{\alpha}
$$

with $\alpha=0,1 \ldots, n-1$. One easily verifies that for either choice of sign, one has as many solutions as in flat space. 3. Because $A d S_{n}$ is conformally flat the Killing spinors in fact satisfy the conformally invariant equation

$$
\nabla_{\alpha} \gamma_{\beta} \epsilon+\nabla_{\beta} \gamma_{\alpha} \epsilon=\frac{1}{2 n} g_{\alpha \beta} \nabla^{\sigma} \gamma_{\sigma} \epsilon
$$

which forms much of the basis of "Twistor theory". Conformal Killing spinors of course arise naturally in conformal supergravity [16]. As a further illustration of historical antecedents, it is interesting to recall that the existence of solutions

\footnotetext{
${ }^{3}$ Using the isometric embedding of $A d S$ as an affine quadric that we shall be describing in detail later, the solutions are easily exhibited as the restriction to the quadric of constant spinors in the flat embedding spacetime.
} 
to an equation of the form (2) was the basic assumption behind the theory of "Wave Geometry" which was extensively developed in Hiroshima in the '30.s. The introduction to [38] describing the history of these ideas and the fate of those working on them seems to me to be one the most poignant in the physics literature.

\subsection{Three-branes and Cosmology}

In what follows we shall mainly be interested in three-branes. This is partly because they connect with results in four-dimensional quantum field theory. However there is an old tradition of speculation which considers our universe as a three-brane moving in some higher dimensional spacetime (see for example [50]) . Recently this idea has been revived [?]. Cosmologists reading this are cautioned therefore against gratuitously assuming that $p$-branes have no relevance for their real world.

\section{The D-three-brane}

Now if $N$ gets large the supergravity approximation should get better and better. Consider the case of $\mathrm{N}$ three-branes, with $N$ large. This has a supergravity description as a classical BPS spacetime solution of the ten-dimensional Type IIB supergravity theory admitting 16, i.e. half the maximum, Majorana-Weyl, that is real, Killing spinors $\epsilon 4$.

\subsection{The Classical Solution}

In isotropic coordinates, which are valid only outside the horizon, the solution takes the form

$$
d s^{2}=H^{-\frac{1}{2}}\left(-d t^{2}+d \mathbf{x}^{2}\right)+H^{\frac{1}{2}} d \mathbf{y}^{2}
$$

where $\mathbf{x} \in \mathbb{E}^{3}$ is a three vector and $\mathbf{y} \in \mathbb{E}^{6}$ is a six vector. $H(\mathbf{y})$ is a harmonic function on $\mathbb{E}^{6}$ and there is also a self-dual five-form

$$
F_{5}=\star F_{5}=d t \wedge d x^{1} \wedge d x^{2} \wedge d x^{3} \wedge d\left(\frac{1}{H}\right)+\star(\text { ditto }) .
$$

\footnotetext{
${ }^{4}$ The reader unfamiliar with supersymmetry but willing to accept that eleven-dimensional physics is behind everything may find it helpful to recall that there are two inequivalent Clifford algebras Cliff $(10,1)$ each isomorphic to $\mathbb{R}(32)$, the algebra of real 32 by 32 matrices, where one may picks the Clifford representative of the volume form $\gamma_{0} \gamma_{1} \ldots \gamma_{10}= \pm$. Let us settle on the plus sign. The matrices $\gamma_{0}, \gamma_{i}, \ldots, \gamma_{9}$ generate Cliff $(9,1)$ and one may split the 32 dimensional space $S$ of Majorana spinors into a direct sum $S=S_{-} \oplus S_{+}$of 16 dimensional positive and negative eigenstates of the Clifford representative $\gamma_{1} 0=\gamma_{0} \gamma_{1} \ldots \gamma_{9}$ of the tendimensional volume form. Elements of the summands are called positive or negative chirality Majorana-Weyl spinors. The student with an interest in global matters is invited to reflect on the remarkable effectiveness of this simple piece of mathematics, once one has made the choice of spacetime signature $(10,1)$, and what it implies for spacetimes lacking space or time orientation and what further things it might betoken for mankind. Guidance for the perplexed may be found in [54].
} 
The dilaton $\phi$ is constant

$$
e^{2 \phi}=g_{s}
$$

If Yang-Mills fields were present the Yang-Mills coupling constant $g_{\mathrm{YM}}$ would be given by

$$
g_{s}=\frac{g_{\mathrm{YM}}^{2}}{4 \pi} .
$$

For a solution representing $N$ three-branes located at positions $\mathbf{y}_{i}, i=$ $1, \ldots, N$, each carrying one unit of 5 -form magnetic flux one chooses

$$
H=1+\sum \frac{4 \pi g_{s} \alpha^{\prime}}{\left|\mathbf{y}-\mathbf{y}_{i}\right|^{4}}
$$

where $\alpha^{\prime}=l_{s}^{2}$ is the Regge slope parameter of string theory and is related to the fundamental string length $l_{s}$.

Now let the $N$ branes coalesce. We get

$$
H=1+\left(\frac{R}{r}\right)^{4},
$$

with

$$
R=\left(g_{\mathrm{YM}}^{2} N\right)^{\frac{1}{4}} l_{s},
$$

and $r=|\mathbf{y}|$.The classical solution is expected to be a good approximation in the limit that $N$ is large but with $\lambda=g_{\mathrm{YM}}^{2} N$ held fixed. This corresponds in $U(N)$ gauge theory to a limit whose study was pioneered by t'Hooft.

\subsection{Near Horizon Geometry}

Isotropic coordinates break down near the horizon at $r=0$. For small $r$ the metric tends to

$$
\left(\frac{r}{R}\right)^{2}\left(-d t^{2}+d \mathbf{x}^{2}\right)+\frac{R^{2} d r^{2}}{r^{2}}+R^{2} d \Omega_{5}^{2},
$$

where $d \Omega_{5}^{2}$ is the standard round metric on $S^{5}$ with unit radius.

Now set

$$
z=\frac{R}{r}
$$

and recall that the standard $A d S_{p+2}$ metric of unit radius in horospheric coordinates $\left(z, x^{\mu}\right)$ is given by

$$
d s^{2}=\frac{1}{z^{2}}\left(d z^{2}+\eta_{\mu \nu} d x^{\mu} d x^{\nu}\right)
$$

with $\mu=0,1, \ldots, p$ and $\eta_{\mu \nu}$ is the Minkowski metric. We deduce that the near horizon geometry is that of $A d S_{5} \times S^{5}$ with the two radii of curvature equal. Taking out $\frac{1}{z^{2}}$ as an overall conformal factor of the limiting ten-dimensional product metric also reveals that it is conformally flat. In fact one may easily extend the argument to show that the metric product of $A d S_{r} \times S^{s}$ with radii $R_{1}$ and $R_{2}$ is conformally flat iff the the two radii of curvature are equal.

Clearly there are considerable advantages associated with horospheric coordinates and we shall be exploiting them further shortly. Before doing so we make a few comments about supersymmetry. 


\subsection{Supersymmetries}

Because it admits a Killing spinor the solution also admits an everywhere causal Killing vector field $K^{\mu}=\bar{\epsilon} \gamma^{\mu} \epsilon$. In fact the solution has the symmetries expected of a three-brane. The isometry group is $E(3,1) \times S O(6)$ with orbits $\mathbb{E}^{3,1} \times S^{5} 5$. In particular it is locally static, but has degenerate Killing horizons. Near infinity the solution tends to flat ten-dimensional Minkowski spacetime $\mathbb{E}^{9,1}$ which clearly admits the maximum possible, i.e. 32 Majorana-Weyl Killing spinors. Near the horizon the spatial sections have an infinitely long throat resembling that of the familiar extreme Reissner-Nordstrom solution. The solution tends, as we have seen, to the product metric on $A d S_{5} \times S^{5}$, with the two radii of curvature having equal magnitude. This solution is also admits 32 Majorana-Weyl Killing spinors and is thus a maximally supersymmetic ground state of type IIB supergravity theory. In fact it is the basis of a "spontaneous compactification" in which one obtains an effective five-dimensional supersymmetric maximally superysmmetric ground state which is geometrically given by $A d S_{5}$. Fluctuations around this solution are given, at the supergravity level, by a five-dimensional gauged supergravity model with gauge group $S O(6)$. Such theories and the properties of such vacua were intensively studied in the past, using just the extensions I alluded to above of Poincaré covariant quantum field theory to the Anti-de-Sitter setting. In the past, the case of $A d S_{4}$, usually times $S^{7}$ or some other compact seven-dimensional Einstein manifold with positive scalar curvature was of greatest physical interest. However the lessons learnt then readily generalize.

Remarkably however, quite unlike the extreme Reissner-Nordstrom solution, the three brane solution is geodesically complete and everywhere non-singular 33 .

\subsection{Vacuum Interpolation, Conformal Flatness and Couch- Torrence symmetry}

This phenomenon is referred to as Vacuum Interpolation [17].It is a feature of many other examples. For example the M2-brane of eleven dimensions spatially interpolates between $\mathbb{E}^{10,1}$ and $A d S_{4}$ times $S^{7}$ and the M5-brane of eleven dimensions spatially interpolates between $\mathbb{E}^{10,1}$ and $A d S_{7}$ times $S^{4}$. They both admit 16 Killing spinors but only the latter is everywhere singularity free. The former has singularities very similar to those of Extreme Reissner-Nordstrom. However neither has another very striking feature of the D3-brane, which it

\footnotetext{
${ }^{5}$ During the sixties there was an intensive, purely group theoretic, discussion of the possibility of combining spacetime, $E(3,1)$ and internal Lie group symmetries in some unifying non-compact group $G$ [4]. The upshot was various No-Go Theorems such as those of McGlinn, O'Raifeartaigh and Coleman and Mandula telling one essentially only to consider the direct product of the Poincaré group and a compact semi-simple group. This is of course typically what results from Kaluza-Klein theory and other dimensional reduction schemes. It might be interesting to revisit those old ideas in the M-Theory context to see if anything more can be said, given some that the group $G$ must act on a higher dimensional spacetime as an isometry group.
} 
shares with the extreme Reissner Nordstrom solution (RN) is that, because in that case the radii of curvature of the two factors are equal, the metric is conformally flat and has vanishing Weyl tensor. For the M2 and M5 brane, the radii are different and this is not so.

In fact both the D3 and the RN admit an involution which acts by conformal isometries and interchanges the horizon and infinity. For the three-brane the involution is given by

$$
r \rightarrow \frac{R^{2}}{r}
$$

under which

$$
d s^{2} \rightarrow\left(\frac{R}{r}\right)^{2} d s^{2} .
$$

I first became aware of this symmetry from a paper of Couch and Torrence in the Reissner-Nordstrom case 4], hence the name I have given its natural generalization. In Schwarzschild coordinates $r$ in an RN solution of mass M the involution is given by

$$
r-M \rightarrow \frac{M^{2}}{r-M}
$$

Of course the isotropic coordinate $|\mathbf{y}|=r-M$ in this case.

It remains unclear whether this symmetry will turn out to play a bigger role in the theory. In other words how, if at all, does this symmetry manifest itself in the quantum theory?

\section{$4 \quad A d S_{p+2}$ and its Horospheres}

The standard definition of $A d S_{p+2}$ is as the quadric $M$ in $\mathbb{E}^{p+1,2}$ with its induced Lorentzian metric given by

$$
\left(X^{0}\right)^{2}+\left(X^{p+2}\right)^{2}-\left(X^{1}\right)^{2}-\left(X^{2}\right)^{2}-, \ldots-\left(X^{p+1}\right)^{2}=1 .
$$

Topologically $A d S_{p+2} \equiv \mathbb{R}^{p+1} \times S^{1}$, and the isometry group is $O(p+1,2)$. Later we shall describe the universal covering spacetime $A \tilde{d} S_{p+1}$.

We remark here that $A d S_{p+2}$ has a natural complexification $M_{\mathbb{C}} \equiv S O(p+$ $3 ; \mathbb{C}) / S O(p+2 ; \mathbb{C})$ as a complex affine quadric

$$
(A+i B)^{2}=1,
$$

with $A+i B \in \mathbb{C}^{p+3}=\mathbb{R}^{p+3}+i \mathbb{R}^{p+3}$ in which $A d S_{p+2}$ sits as a real section with $B^{1}=B^{p+3}=A^{1}=\ldots A^{p+1}=0$ and $A^{1}=X^{0}, A^{p+3}=X^{p+2}, B^{1}=$ $X^{1}, \ldots, B^{p+2}=X^{p+1}$. Of course the complexification contains other real sections. What is usually called the "Euclidean section of $A d S_{p+2}$ " is another real section of $M_{\mathbb{C}}$ for which $X^{0}$ is pure imaginary and the remaining coordinates are real . This gives hyperbolic space $H^{p+2}$. For more details about complexified spacetimes and real slices the reader is referred to [1].

Considered as a real $(2 p+4)$-dimensional manifold $M_{\mathbb{C}} \equiv T S^{p+2}$, the tangent bundle of the $(p+2)$-sphere. This will be explained in detail later. 
To return to $A d S_{p+2}$, the $\mathbb{Z}_{2}$ centre of the isometry group is generated the antipodal map. This is the involution

$$
J: X \rightarrow-X
$$

By definition $J^{2}=$ id. Even though it admits CTC's and indeed closed timelike geodesics (CTG's) nevertheless $A d S_{p+2}$ is time orientable (by deeming that anti-clockwise motion in $X^{0}-X^{p+3}$ is towards the future for example) and the involution $J$ preserves the time orientation. Anti-de-Sitter spacetime is also space-orientable. If $p$ is even then $J$ does not preserve space orientation but if $p$ is odd then it does. Now if $p>1$ then $O(p+1,2)$ has four connected components. If $p$ is odd then the centre $J$ lies in the component connected to the identity. If $p$ is even then it does not. Thus in the odd case, unless one has good reason, one might expect $J$ to be a gauge symmetry of the theory and one might expect to be able to or to be forced to quotient by $J$. This is sometimes referred to as the Elliptic Interpretation. It would amount to spacetime being the quotient $A d S_{p+2} / J$. If $p$ is even then the quotient will not be space orientable. If $p$ is odd then it will 6 . In any event the way that the quantum representative of $J$

$$
\hat{J}: \mathcal{H}_{\mathrm{qm}} \rightarrow \mathcal{H}_{\mathrm{qm}}
$$

acts on the quantum mechanical Hilbert space $\mathcal{H}_{\mathrm{qm}}$ is clearly of considerable interest.

Note that exactly parallel remarks apply to the so-called "R-symmetry" group $O(6)$. Total inversion lies in the identity component $S O(6)$ and taking the quotient gives the orientable five-manifold $\mathbb{R P}^{5}=S^{5} / \pm 1$.

Horospheric coordinates $\left(z, x^{\mu}\right)$ are defined by

$$
X^{0}+X^{p+1}=\frac{1}{z}
$$

and

$$
X^{\mu}=\frac{x^{\mu}}{z}
$$

with $\mu=0,1, \ldots, p$.

The horospheres are given by $z=$ constant. Each one has the intrinsic geometry of $p+1$ dimensional Minkowski spacetime, just like a flat $p$-brane. In fact we have a a foliation of $A d S_{p+2}$ by "test" $p$-branes each one of which is the intersection of the quadric with a null hyperplane in $\mathbb{E}^{p+1,2}$. By $O(p+1,2)$ symmetry is is easy to see that each horosphere is totally umbilic In fact if $p=3$ one may check that each horosphere solves the equation of motion for a test or "probe" D3-brane in this supergravity background, including so-called "Wess-Zumino" terms. Moreover the same is true for the the $r=$ const surfaces in the exact D3-brane metric.

\footnotetext{
6 In the case of $d S_{p+2}$ the analogue $J$ always reverses time orientation. Passage to the quotient is then disastrous because one is forced to real quantum mechanics [?]
} 
This gives a rather graphic illustration of how one may think of the solutions as being the result of the superposition if a very large number of light threebranes.

Since

$$
J:\left(z, x^{\mu}\right) \rightarrow\left(-z, x^{\mu}\right)
$$

we need both positive and negative $z$ patches to cover all of $A d S_{p+2}$. The patches are separated by a Killing horizon at $z=\infty$ which gives rise to a coordinate singularity which is simply the intersection of the quadric with a null hyperplane passing through the origin. later we will provide a more group theoretic description of horospheres.

\subsection{Extension of the full three-brane metric}

This is most simply done [33] by defining

$$
z^{4}=H=1+\left(\frac{R}{r}\right)^{4} .
$$

Thus

$$
\frac{r}{R}=\left(z^{4}-1\right)^{-\frac{1}{4}}
$$

The metric becomes

$$
d s^{2}=\frac{R^{2}}{z^{2}}\left(-d t^{2}+d \mathbf{x}^{2}\right)+\frac{R^{2}(d z)^{2} z^{6}}{\left(z^{4}-1\right)^{\frac{10}{4}}}+\frac{R^{2} z^{2}}{\left(z^{4}-1\right)^{\frac{1}{2}}} d \Omega_{5}^{2} .
$$

This is clearly even in $z$ and the horizon is at $z=-\infty$ but now spatial infinity corresponds to $z= \pm 1$. Using the embedding formula, one may push the exact three-brane metric onto the Anti-de-Sitter metric to give an embedding of the three-brane metric as the proper-subset of $A d S_{5} \times S^{5}$ given by $z^{2}>1$. One may check that $z=1$ corresponds to a conformal boundary with two connected components analogous to the "Scri" of an asymptotically flat black hole. The entire setup is invariant under the action of anti-podal map $J$. One may therefore if one chooses quotient by $J$ to get a three-brane whose outside and inside are the same!

\section{Covering Spaces, the Eternal Return and Wrap- ping in Time}

Many physicists are unhappy with the CTC's in $A d S_{p+2}$ and seek to assuage their feelings of guilt by claiming to pass to the universal covering spacetime $A \tilde{d} S_{p+2}$. In this way they feel that they have exorcised the demon of "acausality". However, therapeutic uttering these words may be, nothing is actually gained in this way. Consider for example the behaviour of test particles. Every timelike geodesic on $A d S_{p+2}$ is a closed curve of the same durations equal to $2 \pi R$, which Heraclitus would have called the "Great Year". 
In fact all geodesics which depart from a particular event meet up again at the same event after six Great Months. To see this we write the metric in Friedman-Lemaitre-Robertson-Walker form. Geometricaly speaking this is a geodesic normal coordinate system. If $X^{0}=\sin t$ and $X^{A}=T^{A} \cos t$, where $T^{0}=0$ is a timelike unit vector , $T^{A} \eta_{A B} T^{B}=-1$, the metric is

$$
d s^{2}=-d t^{2}+\sin ^{2} t d \Omega_{p+1,-1}^{2},
$$

where $d \Omega_{p+1,-1}^{2}$ is the standard metric on $p$-dimensional hyperbolic space $H^{p}$. Each point on on $H^{p}$ corresponds to a timelike geodesic. They all start from one event at $t=0$, reconverge again at $t=\pi$, pass through each other and meet up again in at $t=2 \pi$ and then continue to repeat this cycle for ever. Of course, the metric breaks down at the events $t=\ldots-2 \pi,-\pi, 0, \pi, 2 \pi, \ldots$ but that is because geodesic normal coordinates become singular.

Clearly any observable calculated using timelike geodesics will similarly recur after one Great Year. As far as they are concerned we are effectively on the identified space. Of course we should look carefully at fluctuations about the background and the boundary conditions to see whether we can have any behaviour which does not recur after one great year. We will turn to this point in detail later.

In the meantime we note that if we pass to the universal covering space $\tilde{D} 3$ we may lift the antipodal map and call it $\tilde{J}$. Now $\tilde{J}$ generates an action of the integers taking one asymptotically flat region to infinitely many more. We could, if we wished identify after any number $k$ of actions of $\tilde{J}$. We shall call this spacetime $D 3_{k}$ and we call the act of identification "wrapping in time".

One situation in which wrapping in time may be advantageous is if we want to identify the spatial coordinates of the three-brane, as would be natural if it were wrapped over a non-trivial cycle in a topologically non-trivial spacetime with a torus factor. The problem is that spatial translations do not act freely. They have fixed points on the horizon. These fixed points would give rise to orbifold singularities if one identified under their action. Because $\tilde{J}$ acts freely, these singularities are eliminated if one composes with some power of $\tilde{J}$, in other words as long as one wraps in time as well as in space.

It is important to distinguish between this type of wrapping in time and that obtained by considering the world volume of the three brane as a so-called " discrete spacetime" of the type considered in the elegant construction of Schild 35, 37. In our terms he considers $\Sigma_{4}=\mathbb{E}^{3,1} / L$ where $L$ is the unique Lorentzian self-dual lattice in four dimensions. That model has many attractive features, including invariance under the cover of the discrete Lorentz group $S L(2, \mathbb{G})$ where $\mathbb{G}$ are the Gaussian integers but would, as should be obvious from the discussion above lead to orbifold singularities.

\section{$6 \quad A d S_{p+2}$ as a solvable group manifold}

It is clear from horospheric coordinates that the Poincaré group $E(p, 1)$ acts on $A d S_{p+2}$, but obviously not transitively. The largest orbits are the horo- 
spheres which are the orbits of the $\mathbb{R}^{p+1}$ group of translations. To get a $(p+1)$ dimensional orbits, one must add the $\mathbb{R}_{+}$action referred to for good reasons as the dilatations:

$$
\begin{aligned}
x^{\mu} & \rightarrow \lambda x^{\mu}, \\
z & \rightarrow \lambda z,
\end{aligned}
$$

with $\lambda \in \mathbb{R}_{+}$. The dilatations act on the horospheres. In the embedding space they consist of boosts in the $X^{0}-X^{p+1}$ two plane which take the family of parallel null hyperplanes planes into themselves. but leaving invariant the hyperplane passing through the origin which corresponds to the Killing horizon $z \rightarrow \infty$.

Clearly the $p+2$ dimensional semi-direct product $G_{p+2}=\mathbb{R}_{+} \ltimes \mathbb{R}^{p+1}$ acts simply transitively on one half of $A d S_{p+2}$ [15]. A convenient matrix representation for $g \in G_{p+2}$ is given by thinking of $x^{\mu}$ as a row matrix and mapping

$$
g \rightarrow\left(\begin{array}{cc}
z & x^{\mu} \\
0 & \delta_{\nu}^{\mu}
\end{array}\right)
$$

From this a set of left-invariant Cartan-Maurer one forms is easily seen to be given by

$$
g^{-1} d g=\left(\begin{array}{cc}
z^{-1} d z & z^{-1} d x^{\mu} \\
0 & 0
\end{array}\right)
$$

The $A d S_{p+2}$ metric is clearly left-invariant. Note that since $G_{p+2}$ is not semisimple, the Killing form of $G_{p+1}$ is singular and does not provide a metric.

Note that $G_{p+2}$ is a subgroup of the causality group $\mathbb{R}_{+} \ltimes E(p, 1)$ which, by the Alexandrov-Zeeman theorem [7] [8], is the largest group leaving invariant the causal structure of Minkowski-spacetime $\mathbb{E}^{p, 1}$. It is contained in the conformal group $\operatorname{Conf}(p, 1) \equiv O(p+1,2) / J$ of conformally compactified Minkowski spacetime but contains only those elements of the latter which leave its conformal boundary "Scri", $\mathcal{I}$ setwise invariant.

One could systematically develop the theory of $A d S_{p+2}$ using the left-invariant metric on it $G_{p+2}$ but it seems that this would only give the "outside story" since the orbit of $G_{p+2}$ in $A d S_{p+1}$ contains less than half the space. One can never reach the horizon by acting with the group. Moreover despite the homogeneity of the metric, the group $G_{p+2}$ is geodesically incomplete with respect to the left-invariant metric. In-falling timelike geodesics will penetrate the horizon in finite proper time 7 .

This behaviour is rather reminiscent of ancient discussions of the Edge of the Universe Problem and the No-Boundary Proposal by such cosmologists as Archytas 8 and later Nicholas of Cusa. They argued that the universe cannot have a boundary since if it did, one could always throw a spear towards it. If it had a boundary then the spear must penetrate, leading to a contradiction.

\footnotetext{
7 This is yet another difference that Lorentzian metrics on non-compact group manifolds can bring about compared with Riemannian metrics

${ }^{8} \mathrm{I}$ am grateful to John Barrow for the reference to this Pythagorean from the 5th century BC.
} 
The present example seems to indicate some shortcomings in their logic since, consistent with the homogeneity, the edge of the universe is not actually located at a particular position in $G_{p+2}$. Nevertheless the spear reaches it in finite propertime.

The moral for us today would seem to be that it is more reasonable to adopt a formalism which covers the horizon. Note that restricting to an orbit of $G_{p+2}$ is definitely not the same as adopting the Elliptic interpretation. $A d S_{p+2} / J$, unlike $G_{p+2}$, is geodesically complete. I have never really understood what the slogan "Black Hole Complementarity" means, but possibly this behaviour is an an illustration of what is intended.

The corresponding phenomenon in the case of de-Sitter spacetime is of course the well-known geodesic incompleteness to the past of the Steady State Universe of Bondi,Lyttleton and Hoyle. This may also be thought of as the group manifold $G_{p+1}$. The many attractive features of this model, it's ability to resolve age old philosophical puzzles [25] are due precisely to the group property. The same properties also lead to the physical shortcomings of the model.

\subsection{The Iwasawa decomposition}

We are now in a position to view the horospheres in a more abstract light. Consider, to begin with, a non-compact Riemmanian symmetric space $X=$ $G / H$ where $H$ is the maximal compact subgroup of the simple but non-compact group $G$. Then Iwasawa tells us that any element $g \in G$ may be written uniquely as

$$
g=h a n
$$

where $h \in H, a \in A$ and $n \in N$ where $A$ is abelian and $N$ is nilpotent. The semi-direct product $B=A \ltimes N$ is called the Borel subgroup. That is one may regard the symmetric space $X$ as the group manifold of $B$ equipped with a leftinvariant metric. The orbits of the nilpotent group $N$ are called horospheres. They are labelled uniquely by elements of $A$ and are permuted by elements of $H$.

The basic example is $n$-dimensional hyperbolic space $H^{n} \equiv S O(n, 1) / S O(n)$ which may be regarded as a Wick rotation of $A d S_{n}$ by taking $X^{0}$ to be pure imaginary rather than real. The horospheric coordinate $t$ is then pure imaginary. This is the upper half space model of hyperbolic space, since $z>0$. One has $G=S O(n, 1), H=S O(n), A=\mathbb{R}_{+}$, the dilatations and $N=\mathbb{R}^{p+1}$, the translations. The Iwasawa coordinates are global: they cover all of hyperbolic space.

As we have seen, the case of $A d S_{n}=S O(n, 2) / S O(n .1)$ is similar, except that the Iwasawa coordinates are not global: they do not cover all of $A d S_{n}$. 


\subsection{Symmetric space duality, the Anti-Hopf Fibration and the Goedel viewpoint}

The horosphere concept has a another interesting application to the geometry for $A d S_{n}$ in the case that $n=2 m+1$ is odd. It is illuminating to place the construction in a general context, so we begin by recalling that to every noncompact Riemannian symmetric space $X=G / H$ there is associated a compact symmetric space $\hat{X}=\hat{G} / H$. If the Lie algebra of $G$ is $\mathfrak{g}=\mathfrak{h} \oplus \mathfrak{p}$ then the Lie algebra of $\hat{G}$ is $\hat{\mathfrak{g}}=\mathfrak{h} \oplus i \mathfrak{p}$. Thus the non-compact generators $\mathfrak{p}$ of the non-compact group $G$ have become the compact generators $i \mathfrak{p}$ of the compact group $\hat{G}$. The Riemannian symmetric space $X$ is topologically trivial and carries an Einstein metric with negative scalar curvature. The dual Riemannnian symmetric space is topologically non-trivial and carries an Einstein metric with positive scalar curvature. For example $\hat{H}^{n}=S^{n}$. We can obviously define the inverse map so that for example $\hat{S^{n}}=H^{n}$.

Now choose $\hat{X}=S U(m+1) / U(m) \equiv \mathbb{C P}^{m}$ which is the base manifold of the Hopf fibration of $S^{2 m+1}$ by $S^{1}$,

$$
\mathbb{C P}^{m}=S^{2 m+1} / U(1) .
$$

Explicitly, $S^{2 m+1} \subset \mathbb{C}^{m+1} \equiv \mathbb{E}^{2 m+2}$ is given by

$$
\left|Z^{1}\right|^{2}+\ldots+\left|Z^{m+1}\right|^{2}=1,
$$

where $Z^{a}, a=1, \ldots m+1$ are complex affine coordinates for $\mathbb{C}^{m+1} \equiv \mathbb{E}^{2 m+2}$. The $U(1)$ action is

$$
Z^{a} \rightarrow e^{i \alpha} Z^{a}
$$

Now let us pass to the symmetric space dual of this construction. We replace $S^{2 m+1}$ by $A d S_{2 m+1} \subset \mathbb{C}^{m+1} \equiv \mathbb{E}^{2 m, 2}$ which is given by

$$
-\left|Z^{1}\right|^{2}-\ldots+\left|Z^{m+1}\right|^{2}=1 .
$$

Thus the $U(1)$ action is as before but now it has timelike circular orbits in $A d S_{2 m+1}$, i.e. the orbits are CTC's and therefore the base space has a Riemannian metric. In fact $X=S U(m, 1) / U(m) \equiv H_{\mathbb{C}}^{m}$ is the unit ball in $\mathbb{C}^{m}$ equipped with the Bergman metric, which is the dual of the Fubini-Study metric on $\mathbb{C P}^{m}$. Both are homogeneous Einstein-Kähler 4-metrics, and as such examples of Gravitational Instantons. One has positive cosmological constant and the other has negative cosmological constant. In fact the Bergman metric is the infinite NUT charge limit of the Taub-NUT-Anti-de-Sitter metrics [12.

The metric looks is

$$
d s^{2}=-\left(d t+A_{i} d x^{i}\right)^{2}+g_{i j} d x^{i} d x^{j},
$$

where $i=1,2, \ldots, 2 m, g_{i j}$ is the Einstein-Kähler metric and $d A$ is the Kähler form.

In traditional relativist's language, $A d S_{2 m+1}$ has been exhibited a stationary metric with constant Newtonian potential $U=\frac{1}{2} \log \left(-g_{00}\right)$. The Coriolis 
or gravito-magnetic connection, governing frame-dragging effects corresponds precisely to the connection of the standard circle bundle over the Kähler base space. The curvature is the Kähler form. In fact one may replace the Bergman manifold with any other $2 m$ dimensional Einstein-Kähler manifold with negative scalar curvature and obtain a $(2 m+1)$-dimensional Lorentzian Einstein manifolds admitting Killing spinors in this way.

The general metric is

$$
d s^{2}=-\left(d t+A_{i} d x^{i}\right)^{2}+g_{i j} d x^{i} d x^{j},
$$

where $i=1,2, \ldots, 2 m, g_{i j}$ is the Einstein-Kähler metric and $d A$ is the Kähler form. The timelike coordinate $t$ is periodic with period $2 \pi$. It would seem that there should be applications here to the study of rotation and the AdS/CFT correpondence [30]. A point of interest is that Fourier analyzing the mode QFT mode functions on the spacetime gives rise a to Geometric Quantization problem on the Kähler base manifold. A related construction, not using a Kähler base, providing higher dimensional analogues of the Lorentzian Taub-NUT metric is given in [11.

The simplest case is $m=1$ which is closely related to the Goedel Universe. In this case the base space is two-dimensional real hyperbolic space $H^{2}$ and the Bergman metric is the standard Poincaré metric.

Geometricaly the Goedel universe a product metric on $\mathbb{R} \times S L(\tilde{2}, \mathbb{R})$. For our purposes it is more convenient to pass down to $S L(2, \mathbb{R})$. Now equipped with its bi-invariant or Killing metric one has:

$$
S L(2, \mathbb{R}) \equiv A d S_{3}
$$

and

$$
A d S_{3} / J=S O(2,1) .
$$

In terms of a left invariant basis the bi-invariant metric

$$
d s^{2}=\frac{1}{4}\left(\sigma_{1}^{2}+\sigma_{2}^{2}-\sigma_{0}^{2}\right) .
$$

The anti-Hopf fibres have a time like tangent vector dual to the one-form $\sigma_{0}$.

Goedel himself did not choose the bi-invariant metric but rather a left invariant metric on $S L(\tilde{2}, \mathbb{R})$ which is "locally rotationally symmetric", that is invariant under the right action of $U(1)$. This right action commutes with a left action of a circle subgroup of $S L(2, \mathbb{R})$. His metric is

$$
d s^{2}=\frac{1}{4}\left(\sigma_{1}^{2}+\sigma_{2}^{2}-\lambda^{2} \sigma_{0}^{2}\right)
$$

where $\lambda$ is an appropriately chosen constant so as to solve the Einstein field equations for rigidly rotating dust. Note that $\sigma_{1}^{2}+\sigma_{2}^{2}$ is the standard metric on $H^{2}$. 


\subsection{Heisenberg Horospheres, Finite in all Directions}

If we think of $H_{\mathbb{C}}^{m}$ as the non-compact symmetric space $S U(m, 1) / U(m)$ it also admits a horospherical or Iwasawa decomposition. The abelian factor $A$ is again $\mathbb{R}_{+}$. The nilpotent factor $N$ is now a Heisenberg group [12]. Thus for example , in addition to the standard foliation, $A d S_{5} \equiv U(2,1) / U(2)$ also admits a foliation by a one parameter family consisting of the time-like world volumes of 3-branes. Now because $t$ is periodic these rotating 3-branes have a periodic time coordinate. They are "wrapped in time".

What about "wrapping in space". A related question is whether there is a freely acting discrete subroup $\Gamma \subset S O(n-1,2)$ acting properly discontinuously on $A d S_{n}$ such that $A d S_{n} / \Gamma$ is compact. For reasons connected with the Lorentzian Gauss-Bonnet Theorem, this is only possible if $n=2 m+1$ is odd. In that case there are many suitable $2 m+1$ dimensional lattices $L \subset U(m, 1)$ [20]. Thus indeed one may wrap branes in both space and time in $A d S_{5}$. Moreover, because of the holomorphic nature of the construction, the wrapping should be compatible with superysmmetry.

The resultant non-singular compact Lorentzian spacetimes have no boundary and will certainly have CTC's but may well prove interesting in the context of string theory where compact flat spacetimes have already been analyzed [19]. moreover partially compactified AdS models have already been used to investigate cosmological aspects of the AdS/CFT correspondence [23].

Interestingly, it is an old result of Calabi and Markus that there are no compact quotients of de-Sitter spacetimes without boundary in any dimension. The best one may do is to identify by the antipodal map to get a de-Sitter spacetime with one, rather than the usual past and future boundaries. However, as mentioned earlier, this destroys the time orientation and seems to be fatal quantum mechanically [31].

\subsection{Horospheric Brane-waves}

There is an analogue of the pp-wave metrics which represents gravitational waves propagating in Anti-de-Sitter spacetime which I worked out with Stephen Siklos several years ago (see 43 for details and references). The metrics are conformal

to pp-waves. They may be used to construct $p$-branes on which propagate gravitational waves. Actually the following $(p+2)$ dimensional metric is slightly more general

$$
d s^{2}=\frac{1}{z^{2}}\left\{-d u d v+H\left(u, z, x^{a}\right) d u^{2}+d z^{2}+g_{a b}\left(x^{a}\right) d x^{a} d x^{b}\right\} .
$$

This will satisfy the Einstein equations with cosmological constant as long as

$$
R_{a b}=0
$$

and

$$
z^{p}\left(\frac{\partial}{\partial z}\left(\frac{1}{z^{p}} \frac{\partial H}{\partial z}\right)\right)+\nabla_{g}^{2} H=0
$$


where $a, b=i, 2, \ldots, p-1$ and $\nabla_{g}^{2}$ is the Laplacian with respect to the metric $g_{a b}$. The dependence on $u$ is arbitrary. If the metric $g_{a b}$ is flat, i.e. if $g_{a b}=\delta_{a b}$, then the metric is conformal to a pp-wave. It will then admit half the maximum number of Killing spinors, i.e. those which satisfy

$$
\bar{\epsilon} \gamma^{\mu} \epsilon \frac{\partial}{\partial x^{\mu}}=\frac{\partial}{\partial v}
$$

The right hand side of (46) is a lightlike Killing vector field.

\section{Conformal Compactifications and the bound- ary of $A d S_{p+2}$}

The basic observation behind the AdS/CFT correspondence is the statement that the conformal boundary of $A d S_{p+2}$ is a (two-fold cover of) conformally compactified Minkowski spacetime $\overline{\mathbb{E}^{p, 1}}$. That is

$$
\partial\left(A d S_{p+2}\right)=S^{p} \times S^{1},
$$

or lifting to the universal cover

$$
\partial\left(A d \tilde{S}_{p+2}\right) \equiv E S U_{p+1},
$$

where $E S U_{p+1} \equiv S^{p} \times \mathbb{E}^{0,1}$ is the Einstein static universe. Indeed $\tilde{A} d S_{p+2}$ is conformally flat and may be conformally embedded into one half of $E S U_{p+2}$. It is more or less obvious that the conformal boundary is a copy of $E S U_{p+1}$.

The main idea of Maldacena is that since the isometry group of a manifold, referred to in this context as the "bulk", is the conformal isometry group of its conformal boundary. then Conformal Field Theory on the boundary should, in the large $N$ limit, be equivalent to Type IIB string theory in the interior. The idea is obviously capable of further elaborations and generalizations which I won't enter into here.

We shall start by describing the compactification of Minkowski spacetime and then that of Anti-de-Sitter spacetime.

\subsection{Conformally Compactified Minkowski Spacetime}

If we adjoin to the causality group of $p+1$ dimensional Minkowski spacetime the special conformal transformations

$$
x^{\mu} \rightarrow \frac{x^{\mu}+c^{\mu} x^{2}}{1+2 c_{\mu} x^{\mu}+c^{2} x^{2}}
$$

we obtain the full conformal group $\operatorname{Conf}(p, 1) \equiv S O(p+1,2) / \mathbb{Z}_{2}$. This isomorphism is easily verified at the Lie algebra level but globally things are more subtle. The conformal group acts not on Minkowski spacetime but its conformal compactification $\overline{\mathbb{E}^{p, 1}} \equiv\left(S^{p} \times S^{1}\right) / \mathbb{Z}_{2}$. To see this, we identify $\overline{\mathbb{E}^{p, 1}}$ with the 
space of null rays in $\mathbb{E}^{p+1,2}$. We recover Minkowski spacetime by intersecting with the "light cone" with the null hyperplane

$$
X^{0}+X^{p+1}=\frac{1}{z} .
$$

The stability group of the null hyperplane is just the Poincare group $E(p, 1)$. The null hyperplane captures some but not all of the possible light rays. We miss those parallel to the null hyperplane. These points on the conformal boundary of Minkowski spacetime which is usually called "Scri", standing for script i, $\mathcal{I}$. The entire set of light rays constitute an $\left(S^{p} \times S^{1}\right) / \mathbb{Z}_{2}$.

The usual picture introduced by Penrose is slightly different. It is obtained by regarding the conformal compactification $\{\bar{M}, \bar{g}\}$ of a manifold $\{M, g\}$ as a compact manifold with boundary $\partial \bar{M}$, conformally embedded in some larger manifold $\{\tilde{M} \hat{g}\}$. On $M=\bar{M} \backslash \partial \bar{M} \subset \hat{M}$ one has $\hat{g}=\Omega^{2} g$ where $\Omega$ is a smooth function on $\tilde{M}$ which vanishes on $\partial M$ but such that $d \Omega \neq 0$ on $\partial M$. Thus $\Omega$ vanishes as the distance from the boundary.

Thus Minkowski spacetime in spherical polars has the metric

$$
d s^{2}=-d u d v+r^{2} d \Omega_{p-1}^{2}
$$

where $u-t-r$ and $v=t+r$ are retarded and advanced null coordinates. If we set $u=\tan \left(\frac{T-\chi}{2}\right)$ and $\left.v=\tan \frac{(T+\chi}{2}\right)$ one gets

$$
d s^{2}=\Omega^{-2}\left(d T^{2}+d \chi^{2}+\sin ^{2} d \Omega_{p-1}^{2}\right)
$$

with $\Omega=2 \cos \left(\frac{T-\chi}{2}\right) \cos \left(\frac{T+\chi}{2}\right)$. One sees that

$$
d \Omega_{p}^{2}=d \chi^{2}+\sin ^{2} \chi d \Omega_{p-1}^{2}
$$

is the metric on the unit $p$-sphere with $0 \leq \chi \leq \pi$. Thus The universal cover of the conformal compactification of Minkowski spacetime is the Einstein Static universe $E S U_{p+1} \equiv S^{p} \times \mathbb{E}^{0,1}$. In fact according to a result of Schmidt 64] $E S U_{p+1}$ is maximal in the sense that it cannot be conformally embedded into a a strictly larger manifold. Thus a an open conformally flat $(p+1)$-dimensional manifold ,such as $H^{p} \times \mathbb{E}^{0,1}$ for example, may typically be conformally embedded into $E S U_{p+1}$ as a (possibly proper) subset. This is a standard construction, due to Penrose, for Friedman-Lemaitre-Robertson-Walker universes. We shall use it later when dealing with black holes with exotic topologies.

The involution $\hat{J}$ acts as

$$
\hat{J}:(T, \chi, \mathbf{n}) \rightarrow(T+\pi, \pi-\chi,-\mathbf{n}) .
$$

Thus it consists of a time shift by six Great Months, i.e. half a Great Year, composed with the antipodal map on the $S^{p}$ factor. It therefore identifies what is usually called $\mathcal{I}^{+} \equiv v=\infty \equiv T+\chi=\pi$ with $\mathcal{I}^{-} \equiv u=-\infty \equiv T-\chi=-\pi$. A lightray passing through $\mathcal{I}^{+}$should thus reappear passing through $\mathcal{I}^{-}$.

Of course in the context of conventional macroscopic physics this is ridiculous and clearly does not happen. However there may well be circumstances 
when considering the AdS/CFT correspondence for example, in which the compactified boundary conditions are appropriate.

Consider for example an experimental colleague in the laboratory investigating the steady state configuration of a physical system which is being periodically excited, such as a resonance. The correct boundary conditions for a theorist to use to describe the resonating system are those of the Eternal Return with Great Year equal to to the inverse frequency of the resonance. There is in that case, no question that time "really is" periodic.

In the special case of four-dimensional Minkowski spacetime there is an alternative and some times more useful description (see e,g, 39 for details and references) which starts with thinking of the points $x$ of Minkowsk spacetime as 2 by 2 Hermitian matrices, i.e $x \in u(2)$ the Lie algebra of $U(2)$. The compactification corresponds to passing to the group by means of the the Cayley map

$$
x \rightarrow U=(1+i x)(1-i x)^{-1} .
$$

Thus $\overline{E^{3,1}} \equiv U(2)$. The metric, which is just the obvious invariant metric $-\operatorname{Tr} U^{-1} d U U^{-1} d U$ which is of course Lorentzian. The $U(1)$ factor is timelike. Thus the two fold cover is $S U(2) \times U(1)$ and the universal cover is $S U(2) \times \mathbb{R}$. A similar construction will work for the reals and the quaternions in two and six spacetime dimensions.

In other dimensions there is a related construction using Clifford algebras $x=\gamma_{\mu} x^{\mu}$.

\subsection{The Conformal Compactification of $A d S_{p+3}$}

The embedding of $A d S_{p+1}$ is given by

$$
\begin{gathered}
X^{0}=\sqrt{1+r^{2}} \sin t \\
X^{p+3}=\sqrt{1+r^{2}} \cos t, \\
X^{i}=r \sin \chi n^{i}, \\
X^{p+1}=r \cos \chi .
\end{gathered}
$$

This also gives a conformal embedding into $E S U_{p+2}$ because metric is

$$
\Omega^{-2}\left\{d t^{2}+d \omega^{2}+\sin ^{2} \omega\left(d \chi^{2}+\sin ^{2} \omega d \Omega_{p-1}^{2}\right)\right\}
$$

where $\Omega^{2}=\cos \omega$ and $r=\tan \omega$. Since spatial infinity, $r=\infty$ corresponds to $\omega=\frac{\pi}{2}$ the conformal boundary of $\tilde{A} d S_{p+2}$ is the timelike cylinder $E S U_{p+1}$ as advertized. To get $A d S_{p+2}$ we must identify $t$ modulo $2 \pi$. From (54), it is clear that it's boundary is the two-fold cover of the set of null rays, i.e. of $\overline{\mathbb{E}^{p, 1}}$. The latter is the boundary of $A d S_{p+2} / J$.

Note that if one adopts horospheric coordinates one might have concluded that the conformal boundary of $A d S_{p+2}$ is a copy of Minkowski spacetime $\mathbb{E}^{p, 1}$ situated at $z=0_{+}$. However this is clearly only part of the boundary. Recalling 
that the other side of the horizon has $z$ negative, one might then try to add in another copy situated at $x=0_{-}$. However this leads to overcounting, one must identify points related by inversions

$$
x^{\mu} \rightarrow \frac{x^{\mu}}{x^{2}}
$$

Roughly speaking, one has to attach to Minkowski spacetime the lightcone of the origin. This corresponds to $\mathcal{I}$. However care must be with signs and the upshot is that one lands up on $S^{p} \times S^{p} / \mathbb{Z}_{2}$ again.

\subsection{The Conformal boundary of $H^{p+1}$ and the Doppel- ganger on the other sheet}

Superficially, using the horospheric or upper half space, representation of the metric

$$
d s^{2}=\frac{1}{z^{2}}\left(d z^{2}+d \mathbf{x}_{p+1}^{2}\right),
$$

one might have concluded that the conformal boundary of of of hyperbolic space is $\mathbb{E}^{p+1}$ situated at $z=0$. But this leaves out a single point at $z=\infty$. The boundary is actually $S^{p+1}$. This is most simply seen by thinking of the $H^{p+2}$ as the set of future directed timelike lines passing through the origin of $\mathbb{E}^{p+1,1}$. If one cuts this with a spacelike hyperplane at unit distance the rays are captured inside a ball of unit radius. The bounding $p+1$ sphere corresponds to the null rays through the origin. The detailed calculation is very similar to the standard case of stereographic projection. In spherical coordinates the hyperbolic metric is

$$
d s^{2}=d \omega^{2}+\sinh ^{2} \omega d \Omega_{p+1}^{2} .
$$

If $r=\tanh \left(\frac{\omega}{2}\right)$ this becomes

$$
d s^{2}=\frac{4}{\left(1-r^{2}\right)^{2}}\left(d r^{2}+r^{2} d \Omega_{p+1}^{2}\right) .
$$

One therefore has $\Omega=\frac{1}{2}\left(1-r^{2}\right)$ which vanishes as the distance on the boundary $r=1$.

There is an analogue of the antipodal map for hyperbolic space, reflection in the origin of Minkowski spacetime. However it takes one form the upper sheet of future directed timelike lines to the disconnected lower sheet of past directed timelike lines. One might have thought therefore that the involution plays no role in the "physical sheet". However this is not so. When constructing "Euclidean" Green's functions inside the unit ball one must choose between Dirichlet or Neumann boundary conditions. Calculation reveals that in order to incorporate this it is necessary to add an image source to the direct contribution coming from a Doppelganger on the other sheet and whose strength is equal in magnitude to that of the direct source and whose sign determines whether one has Dirichlet or Neumann case. 
To see this explicitly we first introduce the chordal distance $\sigma\left(x, x^{\prime}\right)$ of two points on $A d S_{p+2}$ or it's complexification

$$
X^{A} \eta_{A B} X^{B}=-1 .
$$

In terms of the embedding coordinates one has:

$$
\sigma=-\frac{1}{2} \eta_{A B}\left(X^{A}-X^{A}\right)\left(X^{B}-X^{\prime B}\right) .
$$

It follows that

$$
\sigma=1+X^{A} \eta_{A B} X^{\prime B} .
$$

Obviously $\sigma=0$ if the points coincide and $\sigma=2$ if they are anti-podal, i.e. $X^{A}=-X^{\prime A}$.

In horospheric coordinates one has

$$
\sigma=\frac{\left(x^{\mu}-x^{\prime \mu}+\left(z-z^{\prime}\right)^{2}\right)^{2}}{2 z z^{\prime}} .
$$

For a scalar field of mass $m$ one defines

$$
\begin{gathered}
a=\frac{p+1}{2}+\sqrt{\left(\frac{p+1}{2}\right)^{2}+m^{2}}, \\
b=\frac{p+1}{2}-\sqrt{\left(\frac{p+1}{2}\right)^{2}+m^{2}} \\
c=\frac{p+1}{2}
\end{gathered}
$$

The free two-point correlation functions may be expressed in terms of hypergeometric functions and, in the Dirichlet case are proportional to

$$
\sigma^{-a} F\left(a, a+1-c, a+1-b ; \frac{2}{\sigma}\right) .
$$

One gets the Neumann case by interchanging the the roles of $a$ and $b$, i.e. taking the opposite sign for the square root in all formulae. The square roots remain positive even if $m^{2}$ is negative, but not too negative. This is the BreitenlohnerFreedman bound

The hypergemetric function has poles at zero, 1 and infinity. The first occurs when the points coincide, the second when they are antipodal. The third when they they have infinite separation.

\section{The Geodesic Flow on AdS and the Future Tube of the boundary}

If one is interested in quantizing a relativistic particle moving in $A d S_{n}$,one approach is to look at the relativistic phase space $T^{\star} A d S_{n}$, pass to the constrained 
space and then to "quantize"it, Because of the high symmetry, one is able to give a rather explicit description of the relevant spaces in group-theoretic terms. They turn out to have some striking properties.

Recall that, in general, the relativistic phase space of a spacetime $M$ is the cotangent bundle $T^{\star} M$ with coordinates $\left\{x^{\mu}, p_{\mu}\right\}$, canonical one-form $p_{\mu} d x^{\mu}$ and symplectic form

$$
\omega=d p_{\mu} \wedge d x^{\mu} .
$$

The geodesic flow is generated by the covariant Hamiltonian

$$
\mathcal{H}=\frac{1}{2} g^{\mu \nu} p_{\mu} p_{\nu}
$$

The flow for a timelike geodesic, corresponding to a particle of mass $m$ lies on the level sets, call them $\Gamma$, given by

$$
\mathcal{H}=-\frac{1}{2} m^{2}
$$

Locally at least, one may pass to the reduced phase space $P=\Gamma / G_{1}$ where $G_{1}$ is the one-parameter group generated by the covariant Hamiltonian $\mathcal{H}$, by a "Marsden-Weinstein reduction". Geometrically, the group $G_{1}$ takes points and there cotangent vectors along the world lines of the timelike geodesics.

The reduced $(2 n-2)$-dimensional phase space $P$ is naturally a symplectic manifold and one may now attempt to implement the geometric quantization programme by "quantizing " $P$.

In the general case it seems to be difficult to carry out this procedure and compare it with the results of more conventional quantum field theory approaches because one does not have a good understanding of the space of timelike geodesics $P$. In the case of $A d S_{n}$ however the space may be described rather explicitly. It turns out to be a Kähler manifold which is isomorphic to the future tube $T_{n-1}^{+}$of $(n-1)$-dimensional Minkowski spacetime.

In $A d S_{n}$ every timelike geodesic is equivalent to every other one under an $S O(n-1,2)$ transformation. They may all be obtained as the intersection of some totally timelike 2-plane passing through the origin of of the embedding space $\mathbb{E}^{n-1,1}$ with the $A d S_{n}$ quadric. The space $P$ of such two planes may thus be identified with the space of geodesics. It is a homogeneous space of the isometry group, in fact it is the Grassmannian $S O(n-1,2) /(S O(2) \times S O(n))$. Note that, as one expects, the dimension of $P$ is $2 n-2$. The denominator of the coset is the maximal compact subgroup of $S O(n-1,2)$. Two factors correspond to timelike rotations in the timelike 2-plane and rotations of the normal space respectively. The former may be identified with the one parameter group $G_{1}$ generated by the covariant Hamiltonian $\mathcal{H}$. Thus the level sets $\Gamma$ is the coset space $S O(n-1,2) / S O(n)$.

Now the striking fact is that the reduced phase space $P \equiv S O(n-1,2) /(S O(2) \times$ $S O(n)$ ) coincides with one of the four series of irreducible bounded symmetric domains, first classified by Cartan [?]. Our case is $\Omega_{n-1}^{I V}$ which, as mentioned above, may also be identified with the Future Tube $T_{n-1}^{+}$of $(n-1$-dimensional 
Minkowski spacetime $\mathbb{E}^{n-1,1}$. This space plays a central role in quantum field theory in flat spacetime since Wightman functions and Green's functions are typically boundary values of holomorphic functions on the future tube. The future tube is defined as those complex vectors $z \in \mathbb{C}^{n-1}$ whose imaginary part lies in the future lightcone.

The space $P$ caries a natural Einstein Khaler metric. The complex structure is given by the $S O(2)$ action. One may regard the Kähler form as the curvature of a circle bundle. This bundle is the constraint manifold $\Gamma$. Actually the entire cotangent bundle $T^{\star} A d S_{n}$, which is a 2 -plane bundle over $P$ carries a Ricci-flat pseudo-Kähler metric. This this metric has signature $(2 n-2,2)$. The timelike coordinates correspond to the time around circle direction, and a coordinate labelling the levels sets $2 \mathcal{H}=-m^{2}$.

The existence of this Ricci-flat pseudo-Kähler metric may be obtained by analytically continuing Stenzels's positive definite Ricci-flat Kähler metric on the cotangent bundle of the standard $n$-sphere, $T^{\star} S^{n}$ 60. The simplest case is when $n=2$. Stenzel's metric is then the Eguchi-Hanson metric which may be analytically continued to give a "Kleinian" metric of signature $(2,1)$ on $T^{\star} A d S_{2}$.

As noted earlier, $T^{\star} S^{n}$ may be identified with an affine quadric in $\mathbb{C}^{n+1}$. This may be seen as follows: $T^{\star} S^{n}$ consist of a pair of real $(n+1)$ vectors $X^{A}$ and $P^{A}$ such that

$$
\begin{gathered}
X^{1} X^{1}+X^{2} X^{2}+\ldots+X^{n+1} X^{n+1}=1, \\
X^{1} P^{1}+X^{2} P^{2}+\ldots+X^{n+1} P^{n+1}=0 .
\end{gathered}
$$

if $P=\sqrt{P^{1} P^{1}+P^{2} P^{2}+\ldots+P^{n+1} P^{n+1}}$ one may map $T^{\star} S^{n}$ into the affine quadric

$$
\left(Z^{1}\right)^{2}+\left(Z^{2}\right)^{2}+\ldots+\left(Z^{n+1}\right)^{2}=1
$$

setting

$$
Z^{A}=A^{A}+i B^{A}=\cosh (P) X^{A}+i \frac{\sinh (P)}{P} P^{A} .
$$

Stenzel then seeks a Kähler potential depending only on the restriction to the quadric (18) of the function

$$
\tau=\left|Z^{1}\right|^{2}+\left|Z^{2}\right|^{2}+\ldots+\left|Z^{n+1}\right|^{2} .
$$

The Monge-Ampère equation now reduces to any ordinary differential equation.

In the case of $A d S_{p+2}$ we may proceed as follows. The bundle of future directed timelike vectors in $A d S_{p+2}, T^{+} A d S_{p+2}$ consists of pairs of timelike vectors $X^{A}, P^{A}$ in $\mathbb{E}^{p+1,2}$ such that

$$
X^{A} X^{B} \eta_{A B}=-1
$$

and

$$
X^{A} P^{B} \eta_{A B}=0
$$


with $P^{A}$ future directed and $\eta_{A B}=\operatorname{diag}(-1,-1,+1, \ldots,+1)$ the metric. We define $P=\sqrt{-P^{A} P^{B} \eta_{A B}}$ and

$$
Z^{A}=\cosh (P) X^{A}+i \frac{\sinh (P)}{P} P^{A}
$$

which maps $T^{+} A d S_{p+2}$ to the affine quadric

$$
Z^{A} Z^{B} \eta_{A B}=-1 .
$$

One then seeks a Kähler potential depending only on the restriction to the quadric (18) of the function

$$
\tau=\left|Z^{0}\right|^{2}+\left|Z^{p+2}\right|^{2}-\left|Z^{1}\right|^{2}-\ldots-\left|Z^{p+1}\right|^{2} .
$$

The Monge-Ampère equation again reduces to any ordinary differential equation.

Let's return to the reduced phase space $P$. It may be realized as a bounded domain $D \subset \mathbb{C}^{n-1}$ and as such it has a $(2 n-1)$-dimensional topological boundary $\partial D$. More interestingly, lying inside this topologically boundary, $\partial D$ is its $(n-)$-dimensional Shilov boundary $S$. If $w \in \mathbb{C}^{n-1}$ is a complex $(n-1)$ column vector and $w^{2}=w^{t} w$ and $|w|^{2}=w^{\dagger} w$ then the domain $D$ is defined by [59]

$$
1-|w|^{2} \geq \sqrt{|w|^{4}-\left|w^{2}\right|^{2}} .
$$

The topological boundary is given by the real equation:

$$
1-2|w|^{2}+\left|w^{2}\right|^{2}=0 .
$$

On the other hand, the Shilov boundary is determined by the property that the maximum modulus of any holomorphic function on $P$ is attained on $S$. Consider, for example, the holomorphic function $w$. It atttains its maximum modulus when $w=\exp (i \theta) \mathbf{n}$, where $\mathbf{n}$ is a real unit $(n-1)$ vector. Thus $S$ is given by $S^{1} \times S^{n-1} / \mathbb{Z}_{2}$.

It is no coincidence that $S$ is topologically the same as the conformal boundary of $A d S_{n}$. To see why, following Hua, who refers to $D$ as "Lie Sphere Space" we can linearise the action of $S O(n-1,2 ; \mathbb{R})$ by embedding $D$ into $\mathbb{C}^{n+1}$. Let

$$
\begin{gathered}
W^{0}-i W^{n+1}=\frac{1}{u}, \\
W^{0}+i W^{n+1}=\frac{w^{2} 2}{u},
\end{gathered}
$$

and

$$
W^{i}=\frac{w^{i}}{u},
$$

where $i=i, \ldots, n-1$ and the complex, horospheric type coordinate $u$ should be set to unity to recover $D$. The $n$ coordinates $\left(u, w^{i}\right)$ Thus parameterize the 
complex lightcone, i.e. the real $2 n$ dimensional submanifold $W \subset \mathbb{C}^{n+1}$ given by.

$$
\left(W^{0}\right)^{2}+\left(W^{n+1}\right)^{2}-W^{2}=0 .
$$

The domain $D$ consists of rays through the origin lying ing in $W$. That is one must identify rays $W^{A}$ and $\lambda W^{A}$, where $\lambda \in \mathbb{C}^{\star} \equiv \mathbb{C} \backslash 0$. Thus $D=W / \mathbb{C}^{\star}$

Evidently $S O(n-1,2 ; \mathbb{R})$ acting in the obvious way on $\mathbb{C}^{n+1}$ leaves $W$ invariant and commutes with the $\mathbb{C}^{\star}$ action. Thus the action of $S O(n-1,2 ; \mathbb{R})$ descends to $D$. If we restrict the coordinates $W^{A}$ to be real we obtain the standard construction of $(n-1)$-dimensional compactified Minkowski spacetime as light rays through the origin of $\mathbb{E}^{n-2,2}$.

The case $n=4$ is special since $S O(4,2) \equiv S U(2,2) / \mathbb{Z}_{2}$. This leads to the equivalence of $\Omega_{2,2}^{I}$ and $\Omega_{4}^{I V}$. As mentioned above, one may identify points in real four-dimensional Minkowski spacetime $\mathbb{E}^{3,1}$ with two by two Hermitian matrices $x=x^{0}+\mathbf{x} \cdot \sigma$. The future tube $T_{4}^{+}$then corresponds to complex matrices $x=z^{0}+\mathbf{z} \cdot \sigma$ whose imaginary part is positive definite. The Cayley map

$$
z \rightarrow w=(z-i)(z+i)^{-1}
$$

maps this into the bounded holomorphic domain in $\mathbb{C}^{4}$ consisting of the space $\Omega_{2,2}^{I}$ of two by two complex matrices $w$ satisfying

$$
1-w w^{\dagger}>0
$$

For more details, the reader is directed to 61. For this approach to the compactification of Minkowski spacetime see also [62, 63.

\section{The Anti-de-Sitter Algebra and Quantized Energies}

If a Lie group $G$ with structure constants $C_{a}{ }^{b}{ }_{c}$ acts on the left on a manifold $M$ the Killing vector fields $\mathbf{K}_{a}$ have Lie brackets

$$
\left[\mathbf{K}_{a}, \mathbf{K}_{c}\right]=-C_{a}{ }^{b}{ }_{c} \mathbf{K}_{b} .
$$

In quantum mechanics one often prefers to work with $\hat{M}_{a}=-i \mathbf{K}_{a}$ acting on spacetime scalar fields is a formally self-adjoint operator with respect to the inner product obtained by integrating over spacetime. Clearly

$$
\left[\hat{M}_{a}, \hat{M}_{c}\right]=i C_{a}{ }^{b}{ }_{c} \hat{M}_{b} .
$$

The $A d S_{p+2}$ group $S O(p+1,2)$ corresponds to

$$
\mathbf{K}_{A B}=X_{A} \partial_{B}-X_{B} \partial_{A},
$$

and therefore

$$
\left[\hat{M}_{A B}, \hat{M}_{C D}\right]=i \hat{M}_{A C} \eta_{B D}-i \hat{M}_{B C} \eta_{A D}+i \hat{M}_{A D} \eta_{B C}-i \hat{M}_{B D} \eta_{A C} .
$$


Upper case Latin indices run form 0 to $p+2$ and $\eta_{A B}=\operatorname{diag}(-1,+1, \ldots,+1,-1)$. Greek indices run from 0 to $p$. Lower case Latin indices run from 1 to $p$.

The maximal compact subgroup of $S O(p+1,2)$ is $S O(p) \times S O(2)$ with generators $\hat{M}_{i j}$ and $\hat{M}_{0, p+2}$. The latter corresponds to rotations in the totally time-like $X^{0} X^{p+2}$ plane. The associated Killing vector field is the globally static Killing field, such that in adapted coordinates the metric is

$$
d s^{2}=-\left(1+r^{2}\right) d t^{2}+\frac{d r^{2}}{1+r^{2}}+r^{2} d \Omega_{p-1}^{2},
$$

with $0 \leq t \leq 2 \pi$.

In the case of $d S_{p+2}$ and $S O(p+2,1), X^{p+2}$ would be spacelike and the maximal compact subgroup would be $S O(p+2)$. It that case $M_{0, p+2}$ would be a non-compact generator corresponding to a boost. The associated Killing vector is not globally static as is clear form the metric in adapted coordinates:

$$
d s^{2}=-\left(1-r^{2}\right) d t^{2}+\frac{d r^{2}}{1-r^{2}}+r^{2} d \Omega_{p-1}^{2},
$$

with $-\infty<t<\infty$. There is a Killing horizon at $r=1$. This difference is crucial for our concept of energy at the classical and the quantum level.

In the De-Sitter case there is no useful global energy concept. As Wigner first realized, there are no "positive energy" presentations of $S O(p+2,1)[29$. The point is that one may easily find a diagonal element of the identity component of $S O(p+2,1)$, call it $g$, such that under the adjoint action

$$
\hat{M}_{p+20} \rightarrow g \hat{M}_{p+20} g^{-1}=-\hat{M}_{p+20} .
$$

The existence of $g$ means that in any unitary representation $\hat{U}(g)$ acts one an energy eigenstate $|E\rangle$ with energy $E$ to give a new state $\hat{U}|E\rangle$ with energy $-E$. Acting on de-Sitter spacetime the element $g$ takes one from one side of the event horizon to the other. This observation is closely related to the thermal emission from cosmological event horizons 36] which Hawking and I discovered in complete ignorance of Wigner's prescient observation.

Wigner's observation is also related to the fact that de-Sitter backgrounds break supersymmetry. Being conformally flat they certainly admit a full set of solutions $\epsilon$ of the twistor equation (3). However the causal vector fields $\bar{\epsilon} \gamma^{\mu} \epsilon$ cannot be Killing vector fields because, as we have seen, there are no everywhere future directed timelike (or null) Killing vector fields on de-Sitter spacetime. In fact the solutions of the twistor equation satisfy

$$
\nabla_{\mu} \epsilon= \pm \frac{i}{2} \gamma_{\mu} \epsilon
$$

A simple calculation reveals however that this equation implies that the causal vector fields $K^{\mu}$ are in fact conformal Killing vector fields.

The situation for Anti-de-Sitter spacetime is completely different. No such element exists for $S O(p+1,2)$ or its universal cover and one does indeed have positive energy representations. One has energy raising and lowering operators

$$
\left[\hat{E}, \hat{M}_{i}^{ \pm}\right]= \pm M_{i}^{ \pm}
$$


with $\hat{M}_{i}^{ \pm}=\hat{M}_{0 i} \pm i \hat{M}_{p+2, i}$ which increase the eigenvalues $E$ by one unit. Thus one finds at the Lie algebra level representations such that the Anti-de-Sitter energy operator has integer spaced eigenvalues:

$$
\hat{M}_{p+20}|E\rangle=E|E\rangle,
$$

with

$$
E=E_{0}+n, n=r, r+1,1 \ldots
$$

with $r$ a non negative integer. The fractional part $E_{0}$ of the energy is constant in each irreducible representation and labels "superselection sectors" 28. If

$$
E=\frac{p}{k}
$$

with $p$ and $k$ relatively prime then we are in fact on the $k$-fold cover of $A d S_{p+2}$. If $E_{0}$ is irrational then we must be on the universal cover. Actually for bosonic fields derived from supergravity fields it turns out that $E_{0}$ vanishes. Thus we can are de facto on $A d S_{p+2}$.

The Poincaré translations are generated by

$$
\hat{P}_{\mu}=\frac{1}{2}\left(\hat{M}_{\mu p+1}+\hat{M}_{\mu p+2}\right) .
$$

The special conformal transformations are generated by

$$
\hat{K}_{\mu}=\frac{1}{2}\left(\hat{M}_{\mu p+1}-\hat{M}_{\mu p+2}\right) \text {. }
$$

The dilatation $D$ corresponds to boosts and is thus given by

$$
\hat{D}=\hat{M}_{p+1 p+2} .
$$

The quantized energy operator is given by

$$
\hat{E}=\hat{M}_{p+20}=\hat{P}^{0}+\hat{K}^{0} .
$$

Now $\hat{E}, \hat{D}$ and $\hat{M}_{0 p+1}$ span an $s l(2 ; \mathbb{R})$ sub-algebra. Thus energy and dilatations do not commute. Hence they cannot be simultaneously diagonalized.

The question of integrality however can be thrown onto the behaviour under the operator $\tilde{\tilde{J}}$.

\subsection{Non-Commutative Coordinates ?}

Of course the generators $\hat{p}_{\mu}=\hat{M}_{p+1 \mu}$ may be thought of as $p+1$ non-commuting "translations" since

$$
\left[\hat{p}_{\mu}, \hat{p}_{\nu}\right]=i \hat{M}_{\mu \nu}
$$

In view of the great current interest in non-commutative geometry it may be worthwhile recalling a very early attempt [?] to extract non-commutative coordinates from the $A d S_{p+2}$ algebra. The idea was to take $\hat{x}_{\mu}=\hat{M}_{p+2 \mu}$ as the "coordinates conjugate to the translations". One has

$$
\left[\hat{x}_{\mu}, \hat{x}_{\nu}\right]=-i \hat{M}_{\mu \nu}
$$


and

$$
\left[\hat{p}_{\mu}, \hat{x}_{\nu}\right]=i \eta_{\mu \nu} \hat{M}_{p+1 p+2} .
$$

In eigenstates of the operator $\hat{D}=\hat{M}_{p+1 p+2}$ we seem to be able to extract a spacetime version of the Heisenberg algebra!. However we certainly do not get a central extension in this way. In retrospect this victory looks a trifle hollow but it is clearly closely related at a formal algebraic level to the Heisenberg Horospheres described earlier. It may indicate how to incorporate these older speculative ideas into the M-theory framework. The reader is referred to 34] for a recent and possibly related discussion.

\section{CFT \& ESU aĺa Luscher and Mack}

These authors 18] start with a conformal field theory on Minkowski spacetime $\mathbb{E}^{p, 1}$ and then Wick rotate with respect to a constant time hyperplane to Euclidean space $\mathbb{E}^{p+1}$. Because the theory is conformally invariant it is assumed to extend to the conformal one-point compactification $S^{p+1}$ on which the conformal group $\operatorname{Conf}(p+1) \equiv S O(p+2,1)$ acts.

We recall that the $k$-point compactification of a complete Riemannian manifold $\{M, g\}$ is a smooth compact Riemannian manifold $\bar{M}$ with metric $\bar{g}$ such that $\bar{M} \backslash\left\{x_{i}\right\}$, where $x_{i}, i=1, \ldots, k$ are the infinity points, is diffeomorphic to $M$ and on $M, \bar{g}=\Omega^{2} g$ and where $\Omega$ is a smooth function on $\bar{M}$ which vanishes at the points $x_{i}$ as one over distance squared. Stereographic projection (certainly known to Ptolemy and probably as far back as Hipparchus around 150 $\mathrm{BC}$ ) provides the compactification in the present case. In spherical coordinates the spherical metric is

$$
d s^{2}=d \omega^{2}+\sin ^{2} \omega d \Omega_{p+1}^{2} .
$$

If $r=\tan \left(\frac{\omega}{2}\right)$ this becomes

$$
d s^{2}=(1+\cos \omega)^{2}\left(d r^{2}+r^{2} d \Omega_{p+1}^{2}\right) .
$$

One therefore has $\Omega=(1+\cos \omega)$ which does indeed vanish like the distance squared as one approaches the infinity point at $\omega=\pi$. There is no $\mathbb{Z}_{2}$ factor here because we may think of compactified conformally $\mathbb{E}^{p+1}$ as the set of future directed null rays through the origin of $\mathbb{E}^{p+1,1}$. The Euclidean special conformal transformations correspond to boots.

Luscher and Mack assume that $S O(p=1,1)$ will act nicely on any "Euclidean" conformal field theory on $S^{p+1}$ and moreover that it will satisfy a version of Osterwalder-Schrader positivity with respect to reflection in an equatorial $p$-sphere. The round metric may be written as

$$
\sin ^{2} \chi\left(d \tau^{2}+d \Omega_{p}^{2}\right),
$$

where

$$
d \tau=\frac{d \chi}{\sin \chi}
$$


The coordinate $\tau$ covers the two-point conformal de-compactification of $S^{p+1}$, the metric product $S^{p} \times \mathbb{E}$. The Osterwalder-Schrader reflection map $\theta$ is given by

$$
\theta: \tau \rightarrow-\tau
$$

and the associated semi-group mapping the upper hemi-sphere $\tau>0$ into itself is given by

$$
\tau \rightarrow \tau+a,
$$

with $a \in \mathbb{R}_{+}$.

The net result is that one Wick rotates back to the Einstein Static Universe $\mathrm{ESU}_{p+1}$ by setting

$$
T=i \tau
$$

Using this data Luscher and Mack are able to show that one may obtain a Lorentzian CFT defined on the Einstein Static Universe, $\mathrm{ESU}_{p+1} \equiv \mathbb{E}^{0,1} \times S^{3}$. There exists a quantum mechanical Hilbert space $\mathcal{H}_{\mathrm{qm}}$ for such on which CFT's on which the universal cover $\tilde{O}(p+1,2)$ acts. As we have seen $E S U_{p+1}$ is the universal cover of the conformal compactification of Minkowski spacetime. The obvious question is whether the theory so defined will descend to the conformal compactification $\overline{\mathbb{E}^{p, 1}} \equiv \mathrm{ESU}_{p+1} / \tilde{J}$ itself or a $k$-fold cover.

The answer given by Luscher and Mack is that in general this is not possible.The existence of non-integer dimensions, with fractional parts unequal,

means that the $(\hat{\widetilde{J}})^{k}$ does not act projectively (i.e. up to a phase) on $\mathcal{H}_{\mathrm{qm}}$ and therefore one cannot project onto the space of invariant states.

Of course for very special CFT's it is not excluded that such projections are possible but this requires very special anomalous dimensions. It is perhaps worth remarking here that the Euclidean approach to quantum field theory on $S^{4}$ adopted by Lusher and Mack is almost identical to that used when one considers quantum fluctuations around an $S^{p}$ universe "born from nothing" in quantum cosmology, cf.[12]. For an example in 2-dimensional CFT see based on the Schottky double of a Riemann surface see citeJ.

\subsection{Superysmmetric Boundary Conditions}

These were first addressed by Breitenlohner and Freedman. They found,in the absence of gravity, that one had two choices. Subsequently Hawking showed that demanding that the supergravity fields satisfy the boundary conditions necessary to permit the existence of an asymptotic Killing spinors giving rise to an asymptotic Anti-de-Sitter superalgebra fixed this ambiguity uniquely. These boundary conditions are essential for the positive mass theorem to work in asymptotically Anti-de-Sitter spacetimes. The boundary conditions imply however that the boundary is invariant under $S O(p+1,2)$. In particular the boundary conditions will enforce periodicity with the Anti-de-Sitter period.

Hawking's original work was in four spacetime dimensions but he has recently generalized it to all relevant dimensions. 


\subsection{Singletons}

One of the remarkable features of the representation theory of the Anti-de-Sitter groups are the singleton and doubleton representations and their supersymmetric extensions. Rather than being connected with quantum field theory in the bulk, they are associated with a conformal field theory on the boundary. The simplest example is a conformally invariant scalar field $\psi$. This occurs as the lowest component of a superfield and has been interpreted as giving the transverse oscillations of the $p$-brane [17, 14.

The equation of motion is

$$
-\nabla^{2} \psi+\frac{p-1}{4 p} R \psi=0
$$

where $R$ is the Ricci scalar of $S^{p} \times S^{1}$.

A simple calculation leads to modes of the form

$$
Y_{l} \exp \left(i\left(l+\frac{p-1}{2}\right) T\right)
$$

where $l$ is a non-negative integer $Y_{l}$ is a spherical harmonic on $S^{p}$ which behaves as $(-1)^{l}$ under the antipodal map on $S^{p}$.

Thus the transverse mode satisfies

$$
\psi(\tilde{J} x)=i^{p-1} \psi(x)
$$

Thus for the D3-brane $p=3$ and the oscillations are invariant under $\tilde{J}^{2}$, for the M5-brane $p=5$ so under $\tilde{J}$ and for the M2-brane $p=2$ under $\tilde{J}^{4}$. This fits in remarkably well with the geometric picture based on the spacetime geometry. It seems that, as far as branes are concerned, Heraclitus may have been right after all!

\section{Finite temperatures and Event Horizons with Exotic Topology}

The idea of thermodynamic equilibrium pre-supposes the existence of a timelike Killing field 9 , Hamiltonian or energy operator $\hat{H}$ and conjugate time variable $t$. One aim is to compute the Gibb's partition function

$$
Z(\beta ; \mathcal{H})=\operatorname{Tr}_{\mathcal{H}} \exp (-\beta \hat{H})
$$

where $\beta$ and $\mathcal{H}_{\mathrm{qm}}$ is the quantum mechanical Hilbert space of the system one is considering.

\footnotetext{
${ }^{9}$ strictly speaking, if only conformally invariant matter is considered, a timelike conformal Killing field may suffice. One may then, modulo conformal anomalies, pass to the conformally related stationary metric. This is important in cosmology, since all FriedmanLemaitre-Robertson-Walker metrics are conformally static.
} 
It follows from the Heisenberg equations of motion and the commutativity or anticommutativity of fields at spacelike separations that the trace projects onto states which are periodic or anti-periodic in imaginary time $\tau=i t$ with period $\beta$. This implies that correlation functions are also periodic or anti-periodic in imaginary time. An amusing example arises when one takes considers globally static coordinates in $A d S_{p+2}$. The finite temperature correlation functions are then periodic in both real and imaginary time. In the case of massless fields, when only poles are present, they may be expressed in terms of elliptic functions [53.

If additional mutually commuting conserved charges $\hat{N}^{i}$ are involved one introduces chemical potentials $\mu_{i}$ and considers

$$
Z\left(\beta, \mu_{i}: \mathcal{H}\right)=\operatorname{Tr}_{\mathcal{H}} \exp \left(-\beta \hat{H}+\beta \mu_{i} \hat{N}^{i}\right) .
$$

If the charges $\hat{H}, \hat{N}^{i}$ generate the Lie algebra $\mathfrak{g}$ of a Lie group $G$ then $Z\left(\beta, \mu_{i}\right.$ : $\mathcal{H})$ is a sort of "character" in the representation of the semi-group element $\exp \left(-\beta \hat{H}+\beta \mu_{i} \hat{N}^{i}\right)$ acting on Euclidean fields. In the case of spacetimes $G$ is a maximally commuting subgroup of the isometry group and the charges $\hat{N}^{i}$ are typically associated with angular momenta or Kaluza-Klein momenta. The chemical potentials $\mu^{i}$ are then interpreted as angular velocities or electrostatic potentials. The Wick rotation of the metric is slightly different in that case. Typically one analytically continues to a complex section of the complexification $M_{\mathbb{C}}$.

\subsection{Three kinds of Static metric}

Depending upon which Killing field we take, we will get a different thermodynamics. Assuming that we maintain $S O(p)$-invariance, there are three natural (locally) static coordinate systems for $A d S_{p+2}$. The associated time translation is a one dimensional subgroup $G_{1} \subset S O(2,1) \subset S O(p+1,2)$ acting on the coordinates say $X^{0}, X^{p+1}, X^{p+2}$ and leaving invariant the coordinates $X^{i}$, $i=1, \ldots, p$. The surfaces of constant time orthogonal to the timelines, i.e. to the orbits of $G_{1}$ in $A d S_{p+1}$, have the intrinsic geometry of hyperbolic space and are the intersections with the quadric of a one parameter family of hyperplanes passing through the origin acted upon by $G_{1}$.

The three possibilities correspond to the three conjugacy classes of one parameter subroups of $S O(2,1)$. They can be labelled by $k=1,0$, and are

- $S O(2)$ rotations in the $X^{0}-X^{p+2}$ two-plane. The hyperplanes $X^{0} / X^{p+2}=$ constant are always timelike. The system is globally static, there are no Killing horizons. Time translations corresponds to $\hat{E}=\hat{M}_{p+20}=\frac{1}{2}=$ $\frac{1}{2}\left(\hat{P}^{0}+\hat{K}^{0}\right)$. The metric is

$$
d s^{2}=-\left(1+r^{2}\right) d t^{2}+\frac{d r^{2}}{1+r^{2}}+r^{2} d \Omega_{p, 1}^{2},
$$

where $d \Omega_{p, 1}^{2}=d \Omega_{p}^{2}$ is the metric on the unit $p$ sphere $S^{p}$. 
- Null rotations. The hyperplanes $X^{0} /\left(X^{p+2}+X^{p+1}\right)=$ constant are always timelike or null. The system is not globally static, there is an extreme Killing horizon ar $r=0$. Time translations correspond to $\hat{P}^{0}=\hat{M}_{p+20}+$ $\hat{M}_{p+10}$. The metric is

$$
d s^{2}=-r^{2} d t^{2}+\frac{d r^{2}}{r^{2}}+r^{2} d \Omega_{p, 0}^{2}
$$

where $d \Omega_{p, 0}^{2}$ is the flat metric on $\mathbb{E}^{p}$.

- Boosts in the $X^{0}-X^{p+1}$ two-plane. The hyperplanes $X^{0} / X^{p+1}=$ constant may be spacelike or timelike: the system is not globally static because there is a non-degenerate Killing horizon ar $r=1$ with unit surface gravity. Time translations correspond to $\hat{M}_{p+10}=\frac{1}{2}\left(\hat{P}^{0}+\hat{K}^{0}\right)$. the metric is

$$
d s^{2}=-\left(r^{2}-1\right) d t^{2}+\frac{d r^{2}}{r^{2}-1}+r^{2} d \Omega_{p,-1}^{2}
$$

where $d \Omega_{p,-1}^{2}$ is the metric on hyperbolic space $H^{p}$.

It is of course possible to make identifications, for example one may convert $\mathbb{E}^{p}$ to a torus $T^{p}$ and $H^{p}$ to a closed hyperbolic manifold. In this way one obtains event horizons with exotic topologies. As stated above, this will lead to orbifold singularities if $k=0$, which corresponds to horospheric coordinates with $z=\frac{1}{r}$. Of course the relation of the coordinates $(t, r)$ etc to the embedding coordinates is different in all three cases.

These three examples can be used to define three kinds of (possibly locally) asymptotically Anti-de-Sitter boundary conditions with an associated concept of ADM mass. Taking out $r^{2}$ as a conformal factor, one sees that the conformal boundaries are the conformally flat manifolds:

- $S^{p} \times S^{1}$

- $\mathbb{E}^{p, 1}$

- $H^{p} \times \mathbb{E}^{0,1}$

. In the last two cases these boundaries are geodesically complete as Lorentzian manifolds but as conformal manifolds they are only subsets of the complete conformal boundary.

The cases $k=1$ and $k=0$ have no natural temperature, so it is possible to consider them at an arbitrary finite temperature $T=\beta^{-1}$. If $k=-1$ one must choose $\beta=2 \pi$. One may pass to imaginary time $\tau=i t$ in the usual way and one gets the metric on hyperbolic space $H^{p}$ which, in the cases $k=1$ and $k=0$, has been identified under the action of the integers generated by $\tau \rightarrow \tau+\beta$. 


\subsection{Tachyonic Black holes}

There are in addition black hole solutions, generalizations of the usual Kottler solution, of the form

$$
d s^{2}=-\left(r^{2}+k+\frac{2 M}{r^{p-1}}\right) d t^{2}+\frac{d r^{2}}{r^{2}+k+\frac{2 M}{r^{p-1}}}+r^{2} \Omega_{p, k}^{2} .
$$

The quantity $M$ is proportional to the ADM mass. If $k=1$ and $k=1$ one finds that if the metric is to be non-singular, in the sense that the singularity at $r=0$ is shielded by an event horizon then $M$ must be non-negative. By contrast if $k=-1$ negative values of $M$ are allowed, as long as they are not too negative.

This fits in both with the AdS/CFT correspondence and with Wigner's observations. On the CFT side, in the case of three-branes, one finds that the Higgs fields of the $N=4$ SUSY Yang-Mills theory have a coupling of the form:

$$
-\frac{1}{12} \operatorname{Tr} R \Phi^{2}
$$

where $R$ is the Ricci scalar of the boundary. In the case of $H^{p} \times \mathbb{E}^{0,1}$, this is negative and the coupling behaves like a tachyonic (i.e. negative mass squared) term. On the the group theory side, it is easy to see that the adjoint action of a rotation of $\pi$ in the $X^{0}-X^{p+2}$, that is an advance of of six Great Months, has the effect of reversing the sign of the relevant energy operator $\hat{M}_{p+10}$.

These remarks also fits with some very old ideas that black holes $p+2$ dimensions in theories without a cosmological constant [42]. If the event horizon geometry is $S^{p}$ rather than $H^{p}$, then the isometry group is $S O(p-1,1) \times \mathbb{R}$ rather than $S O(p) \times \mathbb{R}$. The latter is what Wigner called the little group, i.e. the stability group, of the timelike worldline of an ordinary particle. The latter the little group of the spacelike world line of a tachyon.

\subsection{The Horowitz-Myers Conjecture}

By reversing the role of one of the time and one of the spatial coordinates in the $k=0$ case, Horowitz and Meyers find a black hole for which one of the spatial coordinates must be identified with period $\beta=\frac{4 \pi}{p+1}(2 M)^{\frac{1}{p+1}}$. this defines another boundary condition for which the conformal boundary is $S_{\beta}^{1} \times$ $\mathbb{E}^{p-1} \times \mathbb{E}^{0,1}$. One may also identify points on the $\mathbb{E}^{p-1}$ factor to get a torus $T^{p-1}$. The solution is globally static: it does not have an event horizon. The spatial sections have topology $\mathbb{R} \times T^{p-1}$. Let us call this Horowitz-Meyer version of The Kasner-Kottler spacetime, $\mathrm{HM}_{p+2}$.

One might have thought that $H M_{p+2}$ is an "excitation" of the identified space $A d S_{p+1} / \mathbb{Z}$ where the $\mathbb{Z}$ action is $x^{1} \rightarrow x^{1}+\beta$ in horospheric coordinates. However working out the ADM Mass with respect to $A d S_{p+1} / \mathbb{Z}$ using the methods of [] they find it to be negative!

Thus they lead to conjecture that it is $H M_{p+2}$ which is the true ground state with respect to these boundary conditions and that there is some generalization 
of the positive mass theorem to this setting. This is especially intriguing because $H M_{p+2}$ admits no Killing spinors, ie. it is not BPS.

\section{Concluding Observations}

Having set the global scene, I shall make some observations about the the origin of the $A d S$ geometry.

\subsection{Non-linear Realizations and Spontaneous Symmetry Breaking}

The group manifold viewpoint makes it in some sense almost obvious that in any problem in which some sort of spontaneous breaking of translation and dilatation invariance is involved one can expect to be working on $A d S_{p+2}$. One may identify the coordinates $x^{\mu}$ as the Nambu-Goldstone bosons associated with translation invariance and $\phi=\ln z$ as that associated with dilatation invariance.

To see how, consider to begin with, the the case of the breakdown of a conventional global symmetry group $G$ to an unbroken subgroup $H$. A lowenergy effective lagrangian can be constructed from maps from the world-volume of a $p$-brane to $G / H$. This requires a $G$-invariant metric on $G / H$. One may then construct Noether currents and obtain "current algebras".

For the $p$-brane one includes in $G$ the group of translations transverse to the brane, the other variables being interpreted as additional scalar fields. The standard case of quantum field theory occurs when one has no transverse coordinates. The low energy dynamics of a single soliton defined in $\mathbb{E}^{d}$ is a another special case with $p=0$ except that one typically now has a, possibly curved, "moduli space" $\{M, g\}$ of classical solutions whose coordinates include the positions of the soliton and perhaps some internal degrees of freedom, such as phases or scales. The moduli space will certainly admit the action of the Euclidean group $E(d)$ and the position coordinates are associated with the orbits in $M$ of the translation subgroup. In the case of BPS solitons, one also has multi-moduli spaces $M_{k}$ describing the motion of $k$ solitons. They are not just the products $M \times^{k}$ of the single soliton moduli space but at large soliton separation often tend to a product, and thus include a copy of the configuration space $C_{k}\left(\mathbb{R}^{d}\right) \equiv\left(\mathbb{R}^{d}\right)^{k} / S_{k}$. As far as the low energy dynamics are concerned the solitons move in a non-relativistic Newton-Cartan "spacetime" if the form $M_{k} \times \mathbb{E}^{0}$.

Now all this is very reminiscent of Helmholtz's operational ideas about the physical origin the axions of geometry. By geometry he of course meant noneuclidean space geometry. Being a nineteenth century physicist he not surprisingly based his ideas on the "free mobility of rigid bodies". In effect he regarded space as the coset of possible locations $G / H$ where where $G$ is a six-dimensional Lie group containing $H=S O(3)$ as the group of rotations of a rigid body about a fixed point. The possibilities then reduce to to the triple of symmetric Riemannian spaces with $G=(S O(4), E(3), S O(3,1))$. The first and last are of 
course related by symmetric space duality.

Had Helmholtz known about quantum mechanics he might have proceeded differently but arrived at the same result. He might have assumed the existence of a set of operators or observables whose commutation relations generated the Lie Algebra $\mathfrak{g}$. He would then seek to realize them on some Hilbert space $\mathcal{H}_{r m}$. A simple way for him to do so would be to take $L^{2}\left(G / H, \mu_{g}\right)$, where $\mu_{g}$ is the Riemannian volume element with respect to the invariant metric on $g$. In this way non-euclidean geometry would arise naturally from quantum mechanical principles as a consequence of assumptions about physical systems. Obviously extra degrees of freedom could have been incorporated by passing to a bigger group $G_{\text {unifying }}$, the extra degrees of freedom being interpreted as higher dimensions.

To make this picture compatible with relativity and fit the real world is not easy because we have to incorporate a more sophisticated idea of time into the picture. However some elements are clear. The obvious analogues of $S O(3)$ is $S O(3,1)$ and $S O(4), E(3)$ and $S O(3,1)$ are replaced by and $S O(4,1), E(3,1)$ and $S O(3,2)$. We might begin by replacing quantum mechanics by quantum field theory.

One obvious point of difference with the nineteenth century viewpoint is that for many particles we have no simple analogue of multi-particle spacetimes. This is usually taken care of by second quantization in which everything is thought of as happening in the same spacetime. Of course one may always think of $k$-point bosonic correlation functions as being defined on the $k$-th symmetric power of spacetime, but the geometry is just given by the product metric, unlike the case of the BPS monopole moduli spaces, where it very definitely is not the product metric. Moreover to capture all the information, because it is usually inconsistent to confine attention to a definite number of particles, one consider instead the disjoint union $\sqcup_{k} M^{k} / S_{k}$. There do exist covariant multitime formulations of the classical mechanics of $k$ point particles interacting at a distance but they have no single time, as opposed to multi-time Hamiltonian formulation and they have as yet resisted quantization.

\subsection{Anti-de-Sitter space as a Moduli space}

The idea of spacetimes as moduli spaces is in fact not new. Therefore, before discussing the application of these ideas to string theory, it may prove illuminating to recall some rather old ideas about "Sphere Geometry" which go go back to the nineteenth century in which de-Sitter spaces and their metrics arise naturally.

Consider to begin with, the more familiar case of spheres $S^{d}-1$ in Euclidean space $\mathbb{E}^{d}$. This arises physically in sphere packing problems [55, 56] . Spheres have the the equation

$$
U \mathbf{x}^{2}-2 \mathbf{x} \cdot \mathbf{a}+V=0 .
$$

The centre is at $\frac{\mathbf{a}}{U}$ and the radius $R=\sqrt{\frac{\mathbf{a}^{2}}{U^{2}}-\frac{V}{U}}$. 
The $(d+2)$-tuple $a=(\mathbf{a}, U, V)$ and the $(d+2)$-tuple $\lambda a=(\lambda \mathbf{a}, \lambda U, \lambda V)$, $\lambda \neq 0$ give the same sphere. Moreover the radius will be real and non-vanishing as long as

$$
\mathbf{a}^{2}-U V>0 .
$$

Thus the set of $d-1$ spheres in $\mathbb{E}^{d}$ corresponds to a subset of $\mathbb{R} \mathbb{P}^{d+1}$. If we set $U=a^{p+2}+a^{p+1}$ and $V=a^{p+2}-a^{p+1}$ we will recognize the subset as the set of spacelike directions in $\mathbb{E}^{d=1,2}$, i.e. with de-Sitter spacetime identified under the antipodal map, $D e S_{d+1} / \mathbb{Z}_{2}$. In fact more can be said. We may make use of the freedom to rescale the coefficient $U$ to set

$$
R=U
$$

This means that $V=\frac{\mathbf{a}^{2}}{R}-R$ and hence a sphere $a$ corresponds to the unit spacelike $(d+2)$-vector

$$
a^{A}=\left(\frac{\mathbf{a}}{R}, \frac{1}{R}, \frac{\mathbf{a}^{2}}{R}-R\right) .
$$

Evidently the centre and radius $(\mathbf{a}, R)$ are horospheric coordinates for de-Sitter spacetime.

Now If two spheres $a$ and $a^{\prime}$ intersect, then the angle $\theta$ between them is given by

$$
\cos \theta=\frac{1}{2 R R^{\prime}}\left(R^{2}+{R^{\prime}}^{2}-\left(\mathbf{a}-\mathbf{a}^{\prime}\right)^{2}\right)^{2} .
$$

Clearly

$$
\cos \theta=a^{A} a^{\prime B} \eta_{A B}=\frac{1}{2}\left(2-\left(a^{A}-a^{\prime A}\right)^{2}\right) .
$$

Thus the angle between to spheres, i.e. the conformal structure on the space of spheres, is encoded in the chordal distance, i.e. to the causal structure, of de-Sitter spacetime and vice-versa. Under this correspondence, inversion in a sphere corresponds to reflection in the associated hyperplane. In this way sphere packing problems are related to discrete subgroups of $S O(p+1,1)$ generated by reflections [?, [56. Another application (if $d=2$ ) is to the probability distribution of craters on the moon. The metric of De-Sitter spacetime gives the "fractal", i.e. dilatation and translation invariant measure

$$
\frac{d^{d} \mathbf{a} d R}{R^{d+1}} .
$$

What about Anti-de-Sitter spacetime? We have a similar picture but we must take care with signs. Consider a spacelike hyperbola of two sheets in Minkowski spacetime $\mathbb{E}^{p, 1}$. Its equation is

$$
U x^{2}-2 x^{\mu} a_{\mu}+V=0 .
$$

The central spacetime event is at $\frac{a^{\mu}}{U}$ and we will get a two-sheeted hyperbola as long as

$$
U V-a_{\mu} a^{\mu}>0
$$


This corresponds to $A d S_{p+2} / \mathbb{Z}_{2}$. The "radius" is given by $\sqrt{\frac{\mathbf{a}^{2}}{U^{2}}-\frac{V}{U}}$. In other words the longest proper time between the two sheets is twice the "radius". We may interpret horospheric coordinates of Anti-de-Sitter spacetime as the coordinates of the central event and the size of the hyperbola. If we had chosen to consider the space of single sheeted hyperbolae in Minkowski-spacetime we would have considered spacelike directions in $\mathbb{E} p, 1$ and arrived at a "spacetime" with two times.

\subsection{Twistors and Line Geometry}

The space of spheres or pseudo-spheres carries a natural conformal structure in all dimensions. The case of lines however, in general will not. Plücker and Klein discovered that one may give a conformal structure to the space of lines in $\mathbb{R P}^{3}$. A line in $\mathbb{R P}^{3}$ determines up to scale and a simple bi-vector $\omega \Lambda^{2}(\mathbb{R})$. Two lines $\omega$ and $\omega^{\prime}$ intersect if and only if $\omega \wedge \omega^{\prime}=0$. This quadratic form has signature $(3,3)$ and therefore the set of lines may be identified with the set of null rays in $\mathbb{E}^{3,3}$. This gives $\left(S^{2} \times S^{2}\right) / \mathbb{Z}_{2}$ with metric of signature $(2,1) 10$ Grouptheoretically the projective group $P S L(3 ; \mathbb{R} \equiv S O(3,3)$. Thus if one is prepared to complexify one has a conflation of line geometry and and sphere geometry, that is of the projective geometry of three-dimensions and the and conformal geometry of four-dimensions. This closely related to Penrose's Twistor programme. Any straight line in three dimensions may be lifted to a null geodesic in four-dimensional Minkowski spacetime. Penrose himself prefers to work over the complex but one may restrict one self to some real section and obtain some special cases.

\subsection{Strings in Four Dimensions}

With this set of ideas in mind it is instructive to consider a string theory in four spacetime dimensions. The Nambu-Goldstone modes include four spacetime coordinates. However if If dilatation symmetry is broken one should take the semi-direct product $G_{5}$ of spacetime translations with the dilations. The extra Nambu-Goldstone mode is related to the Liouville mode of string theory. This naturally brings us to consider strings moving in $G_{5}$, i.e. one half of $A d S_{5}$. One might argue that the $S^{5}$ factor has to do with the Goldstone mode for an $S O(6)$ "R" symmetry. This seems to be behind some of Polyakov's thinking about Wilson loops which played an important role in suggesting the AdS/CFT correspondence [?, ?] .

The question the arises: where do the extra generators come to from which are needed to take us behind the horizon ? One possible answer, suggested to me by Tom Banks is as follows. It uses an old result from flat space CFT. Suppose that one has invariance under the Causality Group. Then one should have a canonical energy momentum tensor $T^{\mu}{ }_{\nu}$ which is

\footnotetext{
${ }^{10}$ Because Majorana spinors play such a central role in supersymmetry it may sometimes be useful to recall that the space of projective Majorana spinors for four-dimensional Minkowski spacetime ( with signature $\left(+++-\right.$ ) may be identified with $\mathbb{R P}^{3}$ [57].
} 
- Conserved:

$$
\partial_{\mu} T_{\nu}^{\mu}=0,
$$

- Symmetric

$$
\eta_{\mu \sigma} T^{\sigma}{ }_{\nu}=\eta_{\nu \sigma} T^{\sigma}{ }_{\mu}
$$

and

- Trace-free

$$
T_{\mu}^{\mu}=0 .
$$

Then it follows that one has additional conserved currents coming from the additional conformal Killing vectors associated with special conformal transformations. $K^{\mu}$

$$
\partial\left(T^{\mu}{ }_{\nu} K^{\nu}\right)=0 .
$$

If the boundary conditions permit, one may be able to integrate these over a Cauchy surface to get the missing generators needed to extend the Causality group to the full conformal group. This is essentially the question which was has been addressed at a more rigorous level by Luscher and Mack whose work was desrcibed above.

\section{References}

[1] G W Gibbons and J B Hartle, Real tunneling geometries and the large-scale topology of the universe Phys. Rev. D42 2458-2468 (1990)

[2] E Inönü and E P Wigner, On the Contraction of Groups and Their Representations Proc Natl Acad Sci USA 39 (1953) 510-1953

[3] M Levy-Nahas, Deformations and contractions of Lie Algebras J Math Phys 8 (1967)1211-1222

[4] W E Couch and R J Torrence, Conformal Invariance Under Spatial Inversion of Extreme Reissner Nordstrom Black Holes, Gen Rel Grav 16 (1984) 789-792

[5] G W Gibbons, Aspects of Supergravity Theories, GIFT lectures San Feliu de Guixols, Spain (1984) in Supersymmetry, Supergravity and Related topics eds. F del Aguila, A Azcarraga \& L E Ibanez (World Scientific, Singapore ) (1985)

[6] G C Hegerfeldt and G Hennig, Coupling of Spacetime and Internal Symmetry Fort. der Physik 16 (1968) 491-543

[7] A D Alexandrov, On Lorentz transformations, Uspekhi Mat Nauk 5 (1950) 187

[8] E C Zeeman, Causality Implies Lorentz Invariance, J Math Phys 5 (1964) 490-493 
[9] W L Craig, The Kalām Cosmological Argument, Macmillan (1979)

[10] E R Harrison, Cosmology, Cambridge University Press (1981)

[11] H A Chamblin and G W Gibbons, Topology and Time Reversal, Proceedings of the Erice School on String Gravity and Physics at the Planck Scale, edited by N Sanchez and A Zichichi gr-qc/9510006

[12] G W Gibbons and P Rychenkova, One-sided Domain Walls in M-theory

[13] G W Gibbons, Branes as BIons, hep-the/98030203

[14] G W Gibbons, Wrapping Branes in Space and Time, hep-th/9803206

[15] L Castellani, A Ceresde, R D'Auria, S Ferrara, P Fré and M Trigiante, G/H M-branes and $A d S_{p+2}$ Geometries, hep-th/9803039

[16] M Awada, G W Gibbons and W T Shaw, Conformal Supergravity, Twistors and the Super BMS Group Annals of Physics 171 52-107 (1986)

[17] G W Gibbons and P K Townsend, Vacuum interpolation in supergravity via super p-branes Phys. Rev. Lett. 71 3754-3757 (1994) hep-th/9307049

[18] M L/" uscher and G Mack, Global Conformal Invariance in Quantum Field Theory Comm Math Phys 41 (1975) 203-234

[19] G Moore, Finite in All Directions, hep-th/9305139

[20] A Zeghib, On closed anti de Sitter spacetimes, Math Ann 310 (1998) 695716

[21] G W Gibbons, The Elliptic Interpretation of Black Holes and Quantum Mechanics Nucl. Phys. B271 479-508 (1986)

[22] G W Gibbons and H J Pohle, Complex Numbers, Quantum Mechanics and the Beginning of Time Nucl. Phys. B 410 117-142 (1993) gr-qc/9302002

[23] G T Horowitz and D Marolf, A New approach to String Cosmology, hep-th/9805207

[24] E Calabi and L Markus, Relativistic space forms, Ann Math 75 (1962) 63-76

[25] R S Kulkarni, Proper Actions and Pseudo-Riemannian Space Forms , Advances in Mathematics 40 (1981) 10-51

[26] H S Snyder, Quantized Space-Time Phys Rev 71 (1947) 38-41

[27] H S Snyder, The Electromagnetic Field in Quantized Spacetime Phys Rev 71 (1940) 68-71

[28] E P Wigner, Some Remarks on the Infinite de Sitter Space, Proc Natl Acad Sci USA 36 (1950) 184-188 
[29] T O Phillips and E P Wigner, de Sitter Space and Positive Energy Group Theory and its Applications ed E M Loebl Academic Press NY (1968) 631676 New York (1968)

[30] S W Hawking, C J Hunter and M M Taylor-Robinson, Rotation and the AdS/CFT correspondence, hep-th/9811056

[31] J L Friedman, Lorentzian universes from nothing, Class Quant Grav 15 2639-2644(1988)

[32] L J Alty, The Generalized Gauss-Bonnet-Chern theorem $J$ Math Phys 36 (1995) 3613-3618

[33] G W Gibbons, G T Horowitz and P K Towsend, Higher-dimensional resolution of dilatonic black hole singularities, Class Quant Grav 12 (1995) $297-317$

[34] S Tanaka, Space-Time Quantization and Matrix Model hep-th/9808064

[35] A Schild, Discrete Space-Time and Integral Lorentz Transformations Phys Rev 73 (1948) 414-415

[36] G W Gibbons and S W Hawking, Cosmological Event Horizons, Thermodynamics and Particle Creation Phys. Rev. D15 2738-2751 (1977)

[37] A Schild, Discrete Spacetime and Integral Lorentz Transformations, Canadian J Math 1(1949) 29-47

[38] Y Mimura and H Takeno, Wave Geometry,Scientific Reports of the Research Institute For Theoretical Physics, Hiroshima University 2 (1962)

[39] G W Gibbons and A R Steif, Sphalerons and conformally compactified Minkowski spacetime Phys Lett B346 (1995) 255-261

[40] A Jaffe, S Klimek and A Lesniewski Representations of the Heisenberg Algebra on a Riemann Surface,Comm Math Phys 126 (1989) 421-431

[41] G W Gibbons, C M Hull and N P Warner, The Stability of Gauged Supergravity Nucl. Phys. B218 173-190 (1983)

[42] G W Gibbons and D A Rasheed, Dyson-Pairs and Zero-Mass Black Holes Nucl Phys B 476 515- (1996) hep-th/9604177

[43] G W Gibbons and P J Ruback, Classical Gravitons and their Stability in Higher DimensionsPhys. Lett. B171 390-394 (1986)

[44] G T Horowitz and R Myers, The AdS/CFT correspondence and a new positive energy conjecture for general realtivity hep-th/9808079

[45] Quantum Field Theory in Anti-de Sitter space-time, Phys Rev D18 (1978) 3565 
[46] P Breitenlohner and D Z Freedman Stability in gauged extended supergravity Ann Phys 144 (1982) 249

[47] S W Hawking, The boundary conditions for gauged supergravity, Phys Lett 126B (1983) 175

[48] L F Abbot and S Deser Stability of gravity with a cosmological constant, Nucl Phys B195 (1982) 76

[49] S W Hawking and G T Horowitz, The Gravitational Hamiltonian, action entropy and surface terms Class Quant Grav 13 (1996) 1487-1498 gr-qc/9501014

[50] G W Gibbons and D L Wiltshire, Spacetime as a Membrane in Higher Dimensions Nucl Phys B287 (1987) 717-742

[51] H Nicolai, Representations of Supersymmetry in Anti-de-Sitter Space, in Supersymmetry and Supergravity '84 eds B de Wit and P Van Nieuwenhuizen, World Scientific 1984

[52] L Mezincescu and P K Townsend,Stability at a Local Maximum in Higher Dimensional Anti-deSitter Space and Applications to Supergravity Ann Phys 160(1985) 406-419

[53] B Allen, A Folacci and G W Gibbons, Anti-de Sitter Space at Finite Temperature Phys. Lett. B189 304-310 (1987)

[54] A Chamblin, Existence of Majorana Fermions for M-Branes wrapped in space and time hep-th/9812134

[55] H J Hermann, G Mantica and D Bessis, Space-Filling Bearings, Phys Rev Letts 65 (1990) 3223-3226

[56] D Bessis, Generalized Appollonian Packings, Comm Math Phys 134 (1990) 293-319

[57] G W Gibbons, The Kummer Configuration and the Geometry of Majorana Spinors in Spinors, Twistors, Clifford Algebras and Quantum Deformations eds. Z. Oziewicz et al., Kluwer, Amsterdam (1993)

[58] E Cartan Abh. Math. Sem. Univ. Hamburg 11 (1936) 111-162

[59] L K Hua, Harmonic Analysis of functions of several complex variables in the classical domains Trans Amer Math Soc6 (1963)

[60] M B Stenzel, Ricci-flat metrics on the complexification of a rank one symmetric space, Manusripta Mathematica 80 (19930 151-163

[61] A Uhlmann, Remark on the future tube,Acta Physica Polonica 24 (1963) 293 
[62] A Uhlmann, The closure of Minkowski Space,Acta Physica Polonica 24 (1963) 295-296

[63] G W Gibbons A R Steif, and Sphalerons and Conformally Compactified Minkowski Spacetime Phys. Letts. B 346 255-261 (1995) hep-th/9412210

[64] B G Schmidt, A new definition of conformal and projective infinity of spacetimes, Comm Math Phys 36 (1974) 73-90

[65] P Lounestou and A Springer, Möbius Trsnaformations and Clifford Algebras of Euclidean and Anti-Euclidean Spaces, in Deformations of Strictures (1989) 79-90 ed L Lawrynowicz ed. Kluwer 